BULLETIN (New Series) OF THE

AMERICAN MATHEMATICAL SOCIETY

Volume 38, Number 1, Pages 1-42

S 0273-0979(00)00891-0

Article electronically published on October 18, 2000

\title{
GENERALIZED KAC-MOODY ALGEBRAS AND SOME RELATED TOPICS
}

\author{
URMIE RAY
}

To the memory of my father

Stranger things have happened in the history of physics than the unexpected appearance of sporadic simple groups. [...] We have strong evidence that the creator of the universe loves symmetry, what lovelier symmetry could he find than the symmetry of the Monster?

F. Dyson

\section{INTRODUCTION}

In this review article, we shall give an introduction to the field of generalized KacMoody algebras. This subject gained much interest when Borcherds first proved the remarkable Moonshine Conjecture, which connects two areas apparently far apart: on the one hand, the Monster simple group and on the other elliptic modular functions. Any connection found between an object which has as yet played a limited abstract role and a more fundamental concept is always very fascinating. Also it is not surprising that ideas on which the proof of such a result are based would give rise to many new questions, thus opening up different research directions and finding applications in a wide variety of areas.

We shall start by explaining the Moonshine Conjecture, giving at the same time a brief history of it. We will then give a brief outline of its proof. In the following chapters, we will take one by one the important concepts used in the proof (generalized Kac-Moody algebras, Vertex algebras, automorphic forms), explain them in some detail and mention recent developments connected to them. We shall finish by briefly discussing two interesting topics related to the field of generalized Kac-Moody algebras: Lie superalgebras and lattices.

The author would like to offer her apologies to anyone whose contribution was omitted or not extensively covered. This is not intentional.

Remark. Throughout this paper, the base field will be $\mathbf{C}$.

\section{Contents}

1. The Moonshine Conjecture

1.1. A brief history

1.2. The conjecture

1.3. An outline of the proof

2. Generalized Kac-Moody algebras

\subsection{Definition}

Received by the editors February 8, 2000, and in revised form July 20, 2000, and September $19,2000$.

2000 Mathematics Subject Classification. Primary 17B65, 17 B67.

(C)2000 American Mathematical Society 
2.2. The root system

2.3. Characterizations of a generalized Kac-Moody algebra

2.4. Affine Lie algebras

2.5. Highest weight modules

3. Vertex algebras

3.1. Vertex operators

3.2. Definition of a vertex algebra

3.3. The No-Ghost Theorem and generalized Kac-Moody algebras

3.4. Quantum vertex algebras

3.5. The moonshine module

3.6. The Monster Lie algebra

4. Denominator formulas and automorphic forms

4.1. The denominator formula

4.2. Affine Lie algebras and the Macdonald identities

4.3. The denominator formula of the Monster Lie algebra

4.4. Product expansions of automorphic forms on Grassmannians

4.5. The character formula and Lie algebra homology

4.6. Replicable functions

5. Lie superalgebras

5.1. Definitions and elementary properties

5.2. Character formulas of generalized Kac-Moody superalgebras

6. Lattices

6.1. Some basic facts

6.2. Lattices and modular forms

\section{The Moonshine Conjecture}

1.1. A brief history. The famous Feit-Thompson Odd Order Theorem [FeitT], implying that the only finite simple groups of odd order are the cyclic groups of prime order, sparked off much interest in classifying the finite simple groups in the sixties and seventies. The classification of the building blocks of finite symmetries was completed in the eighties [Gor]: there are 17 infinite families of finite simple groups and 26 which do not belong to any families and are thus called sporadic Bur. The Monster $M$ is the largest sporadic simple group. It has about $10^{54}$ elements or more precisely

$$
2^{46} .3^{20} .5^{9} \cdot 7^{6} .11^{2} .13^{3} \cdot 17 \cdot 19.23 .29 .31 .41 .47 .59 .71
$$

elements. Evidence for the existence of the Monster were first found by B. Fischer and R.L. Griess in 1973. Based on Conway and Norton's conjecture that the dimension of one of its representations is 196883, Fischer, Livingstone and Thorne computed the full character table of $M$ [FisLT]. Then, McKay noticed that the dimensions of its two smallest representations, 1 and 196883, are closely related to the first two coefficients of the Fourier expansions of the normalized modular invariant

$$
J=j-744 .
$$

The modular invariant $j$ is a complex valued holomorphic function on the upper half plane $\mathcal{H}=\{x+i y \mid y>0\}$, which is fixed by the discrete group $S L(2, \mathbf{Z})$ :

$$
\text { for all } \tau \in \mathcal{H}, \quad \text { and } \quad g \in S L(2, \mathbf{Z}), j(g \tau)=j(\tau) \text {. }
$$


We remind the reader that the group $\Gamma=S L(2, \mathbf{Z})$ acts on $\mathcal{H}$ in the following way. Let $a, b, c, d \in \mathbf{Z}$ be integers such that $g=\left(\begin{array}{ll}a & b \\ c & d\end{array}\right) \in \Gamma$. Then for any point $\tau \in \mathcal{H}$,

$$
g \cdot \tau=\frac{a \tau+b}{c \tau+d}
$$

The modular invariant $j$ is meromorphic at infinity and is a bijection from the compactification of $\mathcal{H} / \Gamma, \mathcal{H} \cup\{i \infty\} / \Gamma$ to the compact Riemann sphere $\mathbf{C} \cup\{\infty\}$ such that $j(i \infty)=\infty$. Hence any meromorphic function on $\mathcal{H}$ fixed by $\Gamma$ is a rational function of $j$. Since $j(\tau+1)=j(\tau)$, we can write $j$ as a function of $q=e^{2 \pi i \tau}$. Since $J$ is holomorphic on $\mathcal{H}$, as a function of $q, J$ has a Laurent series in the punctured disc of radius 1 centered at 0 :

$$
J(q)=j(q)-744=q^{-1}+196884 q+\ldots=\sum_{n=-1}^{\infty} c(n) q^{n}
$$

and $c(-1)=1, c(1)=196883+1$. At the time it was thought unlikely that there would be any connection between modular functions and the Monster group. Shortly after, McKay and Thompson found that other coefficients of the $q$-expansion of $J$ are also simple linear combinations of the dimensions of irreducible representations of the Monster group $M$. Now, the dimension of an $M$-module is the trace of the action of the trivial element 1 of $M$ on it, and any $M$-module is completely determined by its character, i.e. the trace function. So based on this observation, McKay and Thompson's insight was to forecast that for all $n \in \mathbf{Z}$ there is an $M$-module $V(n)$ (later called a head representation of $M$ ) of dimension $c(n)$ with character $H_{n}$ such that for all $g \in M$,

$$
T_{g}=\sum_{n=-1}^{\infty} H_{n}(g) q^{n}
$$

would be an interesting function. When $c(n)$ was a known linear combination, the $M$-modules $V(n)$ were to be taken to be the sums of the irreducible representations whose dimensions appeared in the linear combination. As in the case of $V(1)$, the head representations are obviously not just sums of the trivial module!

Thus their work suggested these series, now known as the McKay-Thompson series, were worth investigating. For $g=1$, as already noted, we get the function $J$. Furthermore, they conjectured that $M$ has a natural infinite dimensional representation

$$
V=\oplus_{n \in \mathbf{Z}} V(n)
$$

with graded character $T_{g}$. It came as a surprise that the most natural module of a finite group was infinite dimensional.

1.2. The conjecture. In 1979, Conway and Norton calculated the first 11 coefficients of the McKay-Thompson series for all $g \in M$. Based on these calculations and other observations, they made the following remarkable conjecture [ConN]:

Moonshine Conjecture. For all $g \in M$, the series $T_{g}$ is a normalized Hauptmodul for a genus 0 discrete subgroup of $S L(2, \mathbf{R})$ commensurable with $S L(2, \mathbf{Z})$.

Thus the Moonshine Conjecture reveals a surprising connection between the Monster group (or more precisely its conjugacy classes since characters are class functions) through its head representations and Hauptmoduls. 
We next remind the reader of the definitions and properties necessary to understand the statement of the conjecture. For details, see Shi. Two subgroups, $G$ and $H$ of $S L(2, \mathbf{R})$, are commensurable if both $|H: G \cap H|$ and $|G: G \cap H|$ are finite. For any discrete subgroup $G$ of $S L(2, \mathbf{R})$ commensurable with $S L(2, \mathbf{Z})$, $\mathcal{H}^{*} / G$ is a compact Riemann surface, where $\mathcal{H}^{*}=\mathcal{H} \cup\{$ cusp points of $G\}$. A cusp of $G$ is a point in $\mathbf{R} \cup\{i \infty\}$ fixed by a parabolic subgroup of $G$, i.e. a subgroup with a unique fixed point in $\mathbf{R} \cup\{i \infty\}$.

The group $G$ is said to be of genus 0 if the corresponding Riemann surface is of genus 0 . Then there exist bijections $F$ from $\mathcal{H}^{*} / G$ to the Riemann sphere $\mathbf{C} \cup \infty$, and any meromorphic function on $\mathcal{H}$ fixed by $G$ is a rational function of $F$. If furthermore $F(i \infty)=\infty$, then $F$ is called a Hauptmodul, and in this case there is a smallest positive real number $r$ such that $\left(\begin{array}{ll}1 & r \\ 0 & 1\end{array}\right) \in G$ and so $F(\tau+r)=F(\tau)$. The Hauptmodul $F$ can thus be written as a function of $q=e^{2 \pi i \tau / r}$ and has a Fourier expansion in a punctured disc centered at $0: F(\tau)=\sum_{n \in \mathbf{Z}} a_{n} q^{n}$. As $G$ is of genus $0, F$ has a simple pole at $\infty$. Hence $a_{n}=0$ for all $n \leq-2$. The Hauptmodul $F$ is said to be normalized if $a_{0}=0$ and $a_{-1}=1$.

1.3. An outline of the proof. Conway and Norton not only conjectured that the graded traces $T_{g}$ (for all $g \in M$ ) were Hauptmoduls, but based on the calculation of the first few coefficients, they gave a list of these Hauptmoduls ConN]. So in order to prove the conjecture, one needs to first construct a $\mathbf{Z}$-graded $M$-module (whose existence was conjectured by McKay and Thompson), and then show that the traces are equal to the given Hauptmoduls.

In 1982, Atkin, Fong and Smith found an almost complete computer proof of the Moonshine Conjecture, thus giving strong evidence that the conjecture is correct. In 1983, Frenkel, Lepowsky and Meurman FreLM1 constructed the moonshine module $V$ with graded character given by the Thompson series. In 1992, Borcherds Bor7 gave a very beautiful proof of the Moonshine Conjecture which, together with Frenkel, Lepowsky and Meurman's work, unlike the computer proof, explains how the modular functions and the Monster group are related and leads to new interesting questions and directions of research worth investigating.

The principal ingredients in Borcherds' proof are the moonshine module, vertex algebras and generalized Kac-Moody algebras. Generalized Kac-Moody algebras, also known as Borcherds algebras, are Lie algebras with a root system generalizing the notion of finite dimensional semisimple Lie algebras. They were constructed by Borcherds in 1988 Bor4 and contain as a subclass the symmetrizable Kac-Moody algebras (see $\$ 2$ ), and in particular the affine and finite dimensional simple Lie algebras. The most well known example of a generalized Kac-Moody algebra which is not a Kac-Moody algebra is the Monster Lie algebra, which plays a central role in the proof of the Moonshine Conjecture. In [ConN], Conway and Norton had put forward the idea that there may be a Lie superalgebra which in some way "explains" the Moonshine Conjecture. The construction of the Monster Lie algebra (and thus the proof of the conjecture) is based on the explicit construction by Frenkel, Lepowsky and Meurman [FreLM1] in 1983 (see [FreLM2]) of a Z-graded infinite dimensional vector space fixed by $M$ and of graded dimension $J: V=\sum_{n=0}^{\infty} V(n)$. The module $V$ has a rich algebraic structure.

The first step is to show that $V$ is a vertex algebra with the Virasoro algebra acting on it in an "adequate manner". Vertex algebras were defined by Borcherds 
in 1986 Bor2]. They are an essential part of the proof for the following reason. The construction of an explicit basis for finite dimensional Lie algebras follows directly from its lattice [FreK]. For infinite dimensional generalized Kac-Moody algebras also, explicit bases are known in certain cases [Bor5], [Bor6]. However the construction is no longer direct, and one needs an essential intermediary structure: a vertex

algebra on which the central term of the Virasoro algebra acts by multiplication by a constant at most equal to 26 . As vertex algebras are derived from lattices, this constant depends on the lattice. It has to be unimodular of rank less than 26. This can be shown by applying Goddard and Thorn's No-Ghost Theorem from theoretical physics [GodT]. The generalized Kac-Moody algebra thus derived from the module $V$ is the Monster Lie algebra. Another consequence of the No-Ghost Theorem and of the properties of the moonshine module proved by Frenkel, Lepowsky and Meurman [FreLM1 is that the Monster group $M$ acts on this Lie algebra in such a way that its root spaces are isomorphic to the head representations of $M$.

Now that we know this Lie algebra is a generalized Kac-Moody algebra, we can apply the representation theory of generalized Kac-Moody algebras to it in order to find its denominator formula. This is a formula which contains the essential information on the structure of the generalized Kac-Moody algebra in question. In the case of finite dimensional simple Lie algebras it is the classical Weyl formula, and for affine Lie algebras it is equivalent to the famous Macdonald identities. This was shown by Kac in [Kac2], where a connection between modular functions and infinite dimensional Lie algebras was first discovered. More generally, when this formula is known explicitly it gives an infinite product expansion of an automorphic form. This is basically how generalized Kac-Moody algebras and automorphic forms are related.

The denominator formula for the Monster Lie algebra leads to some further information on its roots, which then allows Borcherds to compute the homology of the Lie subalgebra generated by its positive roots. This calculation shows that for all $g \in M$, the graded traces $T_{g}$ are completely replicable functions [Nor1]. These are modular functions which have in particular the property that all the coefficients of their $q$-expansion can be calculated from the first few. Thus Borcherds completes the proof by showing through a case by case calculation that the traces $T_{g}$ for the action of the Monster on the moonshine module are indeed the Hauptmoduls of the list given by Conway and Norton.

In order to avoid a case by case study, one can use a result of Cummins and Gannon CumG of 1997. Their work explains why the Thompson series are Hauptmoduls and thus makes the last part of Borcherds' earlier proof fully conceptual.

\section{Generalized KaC-Moody algebras}

Before going further, we need to explain what a generalized Kac-Moody algebra is and give some of its characteristics.

2.1. Definition. As mentioned in the introduction, the best way to think of a generalized Kac-Moody algebra $G$ is to consider it as a generalization of a finite dimensional semi-simple Lie algebra. Its definition is based on the Chevalley-Serre construction of finite dimensional semi-simple Lie algebras by generators and relations $[\mathrm{Ser}$. We give Borcherds' original definition Bor4]. 
Let $I$ be a finite set $\{1, \ldots, n\}$ or a countable one identified with $\mathbf{Z}_{+}$, and let $A=\left(a_{i j}\right)_{i, j \in I}$ be a symmetric matrix with real entries satisfying the following two properties:

(i) $a_{i j} \leq 0$ if $i \neq j$,

(ii) $\frac{2 a_{i j}}{a_{i i}} \in \mathbf{Z}$ for all $j \in I$ if $a_{i i}>0$.

Let $H$ be a complex vector space with a symmetric bilinear form (.,.) and elements $h_{i}, i \in I$ for which $\left(h_{i}, h_{j}\right)=a_{i j}$. The elements $h_{i}$ need not be linearly independent.

Then the generalized Kac-Moody algebra associated to the matrix $A$ and with Cartan subalgebra $H$ is the Lie algebra $G$ generated by $H$ and elements $e_{i}, f_{i}, i \in I$ satisfying the following defining relations:

(1) $\quad\left[e_{i}, f_{j}\right]=\delta_{i j} h_{i}$

(2) $\quad\left[h, e_{i}\right]=\left(h, h_{i}\right) e_{i},\left[h, f_{i}\right]=-\left(h, h_{i}\right) f_{i}$

(3) $\quad\left[h, h^{\prime}\right]=0$ for all $h, h^{\prime} \in H$

(4) $\quad\left(\operatorname{ad}\left(e_{i}\right)\right)^{1-\frac{2 a_{i j}}{a_{i i}}} e_{j}=0=\left(\operatorname{ad}\left(f_{i}\right)\right)^{1-\frac{2 a_{i j}}{a_{i i}}} f_{j}$

(5) $\quad\left[e_{i}, e_{j}\right]=0=\left[f_{i}, f_{j}\right]$ if $a_{i j}=0$

The generalized Kac-Moody algebra $G=G(A, H)$ is a Kac-Moody algebra if all the diagonal entries of $A$ are positive. If the indexing set $I$ is finite and the matrix $A$ is positive definite, we recover all the finite dimensional semisimple Lie algebras. If $I$ is finite and the $n \times n$ matrix $A$ is indecomposable of rank $n-1$ and all principal minors of $A$ are positive definite, and $\operatorname{dim} H=n+1$, then we get all the extended affine Lie algebras ([Kac9, chap.4]). We remind the reader that the matrix $A$ is indecomposable if after any reordering of the indices, it can never be written in the form $\left(\begin{array}{cc}A_{1} & 0 \\ 0 & A_{2}\end{array}\right)$.

Note that in the usual definition of a Kac-Moody algebra, and a fortiori of a finite dimensional semisimple Lie algebra, the (generalized) Cartan matrix $A$ is not assumed to be symmetric, but all diagonal entries are equal to 2 . Thus there are Kac-Moody algebras which are not generalized Kac-Moody algebras since they have a non-symmetrizable Cartan matrix. Nevertheless, all the important classes of Kac-Moody algebras (known so far), i.e. finite dimensional semisimple, affine or hyperbolic, have symmetrizable (generalized) Cartan matrix $A$, in other words one which induces a symmetric bilinear form on $H$ in the above manner. By this we mean that for each $i \in I$, there exists $\epsilon_{i}>0$ such that $D A$ is symmetric for $D=\operatorname{diag}\left(\epsilon_{1}, \ldots, \epsilon_{n}\right)$. Since the generators of $G(A, H)$ are multiples of the above given ones, $G(A, H) \cong G(D A, H)$. Therefore when $G$ has a symmetrizable Cartan matrix, it is simpler to assume that $A$ is symmetric. In general, it is hard to prove results for non-symmetrizable Kac-Moody algebras, though Kumar Kum and Mathieu [Mat1 have independently proved a Demazure character formula for irreducible highest modules of arbitrary Kac-Moody algebras.

As mentioned earlier, the elements $h_{i}$ are not in general linearly independent. They need not be distinct even. In many examples of generalized Kac-Moody algebras, for example the Monster Lie algebra, the elements $h_{i}$ are linearly dependent, and so the general setup given above is needed.

For every (generalized) Cartan matrix $A$, there is a unique (up to isomorphism) universal central extension $\tilde{G}(A, \tilde{H})$, which plays an important role in representation theory. Its Cartan subalgebra is generated by elements $h_{i j}$ for $i, j \in I$. When $i \neq j, h_{i j}=\left[e_{i}, e_{j}\right]$ is central in $\tilde{G}$ and $h_{i j} \neq 0$ if and only if $\left(h_{i}, h_{k}\right)=\left(h_{j}, h_{k}\right)$ 
for all $k \in I$. Writing $h_{i}=h_{i i}$, the definition of a generalized Kac-Moody algebra $G$ can be extended to include all semi-direct products of abelian Lie algebras with quotients of $\tilde{G}(A, \tilde{H})$ by central subalgebras contained in $\tilde{H}$ [Bor6]. The abelian Lie algebras correspond to outer derivations having the generators $e_{i}$ and $f_{i}, i \in I$ of $\tilde{G}$ as eigenvectors.

If needed, one can always make sure that the elements $h_{i}$ are linearly independent in $G(A, H)$ by taking an adequate central extension of $G(A, H)$ and adding the necessary number of outer derivations. Up to the size of its centre and its number of outer derivations, the generalized Kac-Moody algebra $G(A, H)$ is uniquely determined by its Cartan matrix $A=\left(\left(\alpha_{i}, \alpha_{j}\right)\right)$ KacP2 .

It is worth mentioning that the above definition of a generalized Kac-Moody algebra by generators and relations generalizes Moody's definition of a Kac-Moody algebra Moo. Kac defined the Kac-Moody algebra $G(A, H)$ as the quotient of the Lie algebra $\tilde{G}(A, H)$ with defining relations $(i)-(i i i)$, by the unique maximal ideal of $\tilde{G}(A, H)$ having trivial intersection with $H$. Using arguments from the representation theory of Kac-Moody algebras, both definitions were shown to be equivalent by Gabber-Kac [GabK]. This implies that a Kac-Moody algebra modulo its centre is simple if its Cartan matrix is indecomposable. Their work generalizes in a straightforward manner to generalized Kac-Moody algebras [Kac9].

2.2. The root system. When the elements $h_{i}$ are linearly independent, the roots can be defined as elements of the dual space $H^{*}$, i.e. as the eigenvalues of the adjoint action of $H$ on $G$. However in the general case, one has to be very careful about the definition of roots. They are not taken to be elements of $H^{*}$, but of the root lattice $Q$ defined as the free abelian group generated by the elements $\alpha_{i}, i \in I$, with a real valued bilinear form given by $\left(\alpha_{i}, \alpha_{j}\right)=a_{i j}$. We shall keep the same notation (.,.) for the bilinear form on $Q, H$ or $H^{*}$. The elements $\alpha_{i}$ are called simple roots.

For $\alpha \in \sum \mathbf{Z}_{+} \alpha_{i}$ (resp. $-\sum \mathbf{Z}_{+} \alpha_{i}$ ), the root space $G_{\alpha}$ is the subspace of $G$ generated by the elements $\left[e_{i_{j}}\left[\ldots\left[e_{i_{2}}, e_{i_{1}}\right]\right]\right]$ (resp. $\left[f_{i_{j}}\left[\ldots\left[f_{i_{2}}, f_{i_{1}}\right]\right]\right]$ ) such that $\sum_{k=1}^{j} \alpha_{i_{k}}=\alpha$. The set of roots $\Delta$ consists of the elements $\alpha \in Q$ for which the space $G_{\alpha}$ is non-trivial. A root $\alpha$ is said to be positive if it is the sum of simple roots. As usual, a root $\alpha \in \Delta$ is negative if and only if $-\alpha$ is a positive root. Hence the set $\Delta$ decomposes into the set $\Delta_{+}$of positive roots and the set $-\Delta_{+}$of negative roots:

$$
\Delta=\Delta_{+} \cup\left(-\Delta_{+}\right)
$$

Furthermore $G$ is $\Delta$-graded:

$$
G=H \oplus\left(\oplus_{\alpha \in \Delta} G_{\alpha}\right) .
$$

Note that if we set $G_{0}=H$, then this is a Lie algebra grading.

There are two types of roots: either a root has positive norm, in which case it is called real, or else it has non-positive norm and it is said to be imaginary. We shall come back to this later.

We often abuse terminology and consider the roots as vectors in $H$ or $H^{*}$ by using the natural map taking $\alpha_{i}$ to $h_{i}$ or to the linear functional $h_{i}^{*}$ in $H^{*}$ mapping $h$ to $\left(h, h_{i}\right)$. We shall do so in this paper. However this map from $\Delta$ to $H$ (respectively to $H^{*}$ ) is not injective when the elements $h_{i}$ (respectively $h_{i}^{*}$ ) are linearly dependent. There may be $n>1$ imaginary simple roots with the same image in $H$ or $H^{*}$ if the 
Cartan matrix $A$ has distinct columns which are equal. In this case, we say that $h_{i}$ (respectively $h_{i}^{*}$ ) has "restricted simple root multiplicity" $n$. Since all non-diagonal entries of $A$ are non-positive, this does not occur for real simple roots. Note that in general, the same vector in $H$ or $H^{*}$ can be the image both of simple and non-simple roots. This happens for a natural class of generalized Kac-Moody subalgebras of the Monster Lie algebra [Ray2. So the "restricted simple root multiplicity" of a root $\alpha$ in $H$ or $H^{*}$ is defined to be the maximal dimension of a subspace of $G_{\alpha}$ which cannot be generated by the root spaces $G_{\alpha_{i}}$, where $i \in I$ such that $\alpha_{i} \neq \alpha$.

In order to avoid this problem, when the elements $h_{i}$ in $H$ or the elements $h_{i}^{*}$ in $H^{*}$ are linearly dependent, there is another standard way of defining roots. Instead of considering the roots as elements of $H^{*}$ (as previously), one extends the Cartan subalgebra to an abelian Lie algebra $H^{e}$ by adding an adequate number of outer derivations. Then the roots are taken to be elements of $\left(H^{e}\right)^{*}$.

We will assume that when the roots are considered to be elements of $H$ or $H^{*}$, the notion of positive and negative roots remains well defined and that there is a Weyl vector $\rho \in H$ defined by

$$
\left(\rho, \alpha_{i}\right)=\frac{1}{2}\left(\alpha_{i}, \alpha_{i}\right) \quad \text { for all } \quad i \in I .
$$

This is the case for the Monster Lie algebra which we shall define later.

The Weyl group of $G$, which controls most of the structure of $G$, is a Coxeter group generated by the reflections corresponding to real simple roots:

$$
W=\left\langle r_{i} \mid\left(\alpha_{i}, \alpha_{i}\right)>0\right\rangle, \quad \text { where } \quad r_{i}: H^{*} \rightarrow H^{*}, r_{i}(\alpha)=\alpha-\frac{2\left(\alpha, \alpha_{i}\right)}{\left(\alpha_{i}, \alpha_{i}\right)} \alpha_{i}
$$

It is worth mentioning that for generalized Kac-Moody algebras with no imaginary simple roots $\alpha_{i}$ orthogonal to all simple roots $\alpha_{j}$ for $j \neq i$, there are three equivalent definitions of a real root $\alpha \in \Delta$ [Kac9]:

(i) $(\alpha, \alpha)>0$;

(ii) $\alpha$ is conjugate under the action of the Weyl group to a simple root of positive norm;

(iii) The $\alpha$-chain through any other root is finite, i.e. $\forall \beta \in \Delta, \beta+n \alpha \in \Delta$ only for finitely many $n \in \mathbf{Z}$.

As we shall see in $\S 6$, one of the problematic features in the more general context of generalized Kac-Moody superalgebras is that this equivalence no longer persists. Note that if for all $j \neq i, a_{i j}=0$ and $a_{i i} \neq 0$, then without loss of generality, the root $\alpha_{i}$ can be assumed to be real since the Cartan matrix can be multiplied by a diagonal matrix $D=\left(d_{i j}\right)$, where $d_{i i}=-1$ and $d_{j j}=1$ for $j \neq i$, to get an isomorphic generalized Kac-Moody algebra.

It follows from definition $($ ii $)$ that real root spaces have dimension 1 and from $(i i)$ and $($ iii $)$ that a root $\alpha$ is real if and only if the Lie subalgebra $G_{\alpha} \oplus G_{-\alpha} \oplus\left[G_{\alpha}, G_{-\alpha}\right]$ is isomorphic to $s l_{2}$ and its adjoint action on $G$ decomposes into finite dimensional representations of $s l_{2}$. None of this may hold for imaginary roots. If all roots are real, $G$ is finite-dimensional. If all the simple roots are real, $G$ is a Kac-Moody algebra. Hence a Kac-Moody algebra is generated by copies of $s l_{2}$, one for each simple root, and the adjoint action of each of these $s l_{2}$ 's on $G$ decomposes into finite dimensional representations. A generalized Kac-Moody algebra is generated by copies of $s l_{2}$ and of the 3-dimensional Heisenberg algebra $(\langle e, f, h=[e, f]\rangle$ with $h$ a central element), and in this case the action of these $s l_{2}$ 's does not necessarily 
decompose into finite dimensional representations. In other words, the difference between a Kac-Moody algebra and a generalized Kac-Moody algebra is that the latter may have imaginary simple roots (corresponding to negative diagonal entries in the Cartan matrix), but they do not add any complication to the structure. Roughly speaking, if no two imaginary simple roots are mutually orthogonal, the imaginary simple roots mostly contribute a free Lie algebra part to $G$ [Kac7, Jur.

Note that generalized Kac-Moody algebras appear naturally in the context of Kac-Moody algebras as the subalgebras of Kac-Moody algebras of elements fixed by finite groups of diagram automorphisms [Bor4].

2.3. Characterizations of a generalized Kac-Moody algebra. As the definition of a generalized Kac-Moody algebra is based on generators and relations, it is difficult to use it to check whether a given Lie algebra is a generalized KacMoody algebra or not. Hence it is important to find other characterizations of these algebras.

If $G=G(A, H)$ is a generalized Kac-Moody algebra, then $\omega: G \rightarrow G$ defined by

$$
\omega\left(e_{i}\right)=-e_{i} \text { and } \omega\left(f_{i}\right)=-f_{i}
$$

is an automorphism of $G$ [Kac9]. Furthermore all generalized Kac-Moody algebras have an invariant symmetric bilinear form (.,.) extending the bilinear form on the Cartan subalgebra and thus generalizing the Killing form. The bilinear form (., .) 0 given by $(x, y)_{0}=-(x, \omega(y))$ is positive definite on root spaces [KacP4]. Based on this, Borcherds gave the following characterization [Bor4. It is essential in the proof of the Moonshine Conjecture, where it is needed to show that the Monster Lie algebra is indeed a generalized Kac-Moody algebra.

Theorem. Let $G$ be a Lie algebra satisfying the following conditions:

(i) $G$ is graded: $G=\sum_{i \in \mathbf{Z}} G_{i}$ with $\operatorname{dim} G_{i}<\infty$ for all $i \neq 0$ and $\left[G_{i}, G_{j}\right]<G_{i+j}$;

(ii) $G$ has an involution $\omega$ of $G$ with $\omega\left(G_{i}\right)=G_{-i}, \omega$ is multiplication by -1 on $G_{0}$;

(iii) there is an almost positive definite Hermitian contravariant bilinear form (.,.) on $G$ : i.e. $([g, x], y)=-(x,[\omega g, y])$ for all $g, x, y \in G$, and $(.,$.$) is positive$ definite on $G_{i}$ if $i \neq 0$. Furthermore $\left(G_{i}, G_{j}\right)=0$ if $i \neq j$.

Then $G$ is a generalized Kac-Moody algebra.

Well known Lie algebras satisfying the conditions of the theorem are direct sums of affine and finite dimensional semi-simple ones, which correspond to the case when the form $(.,$.$) is positive semidefinite on all of G$. Most generalized Kac-Moody algebras satisfy this theorem, though some with infinitely many generators do not Jur. Note that if $G$ satisfies the conditions of the theorem, then this bilinear form is unique up to multiplication by a scalar on the derived algebra $G^{\prime}$ if the Cartan matrix $A$ of $G$ is indecomposable [Kac9].

The above characterization is not always very practical, because for a given Lie algebra there may be many bilinear forms, and it may be complicated to find one with the required properties. In [Bor8], Borcherds gives another characterization in terms of properties of root systems and spaces which is more useful when the Lie algebra is constructed from a lattice. Indeed in this case, it is easier to derive properties of the root system than to find adequate contravariant bilinear forms. Nevertheless searching for other definitions may be worthwhile, as none of the existing characterizations is fully satisfactory. 
2.4. Affine Lie algebras. As explained above, generalized Kac-Moody algebras are a generalization of symmetrizable Kac-Moody algebras, and their structure is essentially the same. In this section, we shall say a few words on the structure of affine Lie algebras since they are the most important representatives of this class of Lie algebras, and also this will be useful both for our discussion of vertex algebras in $\S 3$ and for our illustration of the connection between generalized Kac-Moody algebras and automorphic forms in $\S 5$.

The Lie algebras known today as Kac-Moody algebras were constructed independently by Kac [Kac1] and Moody [Moo] in 1968. The aim of Kac in [Kac1] was to classify all simple graded Lie algebras $G=\sum_{i \in \mathbf{Z}} G_{i}$ of finite growth; i.e. $\operatorname{dim} G_{i}$ is bounded by a polynomial in $|i|$. This classification was completed by Mathieu Mat2 in 1992. Note that in the more general context of Lie superalgebras, this problem is still open.

In Kac1, Kac gives an explicit construction of an important class of graded Lie algebras of finite growth: the affine Lie algebras. It is in this paper and in Moo] that affine Lie algebras appear for the first time in the mathematics literature.

Let $G^{0}$ be a Lie algebra with an invariant symmetric bilinear form $(\cdot, \cdot)$. The affinization of $G^{0}$ is the Lie algebra

$$
G=G^{0} \otimes \mathbf{C}\left[t^{-1}, t\right] \oplus \mathbf{C} c
$$

with

$$
[c, x]=0,\left[a \otimes t^{n}, b \otimes t^{m}\right]=[a, b] \otimes t^{n+m}+(a, b) n \delta_{n+m, 0} c,
$$

for all $x \in G$ and $a, b \in G^{0}$. The element $c$ is called the canonical central element. Let $d$ be the derivation of $G$ acting as follows:

$$
[d, a \otimes f(t)]=a \otimes t \frac{d f(t)}{d t} .
$$

The extended affine Lie algebra $\tilde{G}$ is the semidirect product of $G$ with $\mathbf{C} d$.

When $G^{0}$ is a finite dimensional simple Lie algebra, the Lie algebra $G$ is called an (untwisted) affine Lie algebra [Kac9]. Therefore an affine Lie algebra is a central extension of a loop algebra $G^{0} \otimes \mathbf{C}\left[t^{-1}, t\right]$, where $\mathbf{C}\left[t^{-1}, t\right]$ is the algebra of Laurent polynomials, with an added derivation.

The affine Lie algebras first appeared in the physics literature in 1971 as symmetries of an important class of models of two dimensional conformal field theories. They have other important applications in physics. For example, they play a fundamental role in the analysis of critical phenomena in two dimensional statistical systems. In mathematics they give a framework for the famous Macdonald [Mac] identities for certain powers of the Dedekind $\eta$ function Kac2.

The hyperbolic or Lorentzian Lie algebras form another important subclass of the symmetrizable Kac-Moody algebras. They are defined to be Kac-Moody algebras having a non-degenerate bilinear form (.,.) of signature $n-2$ on the Cartan subalgebra $H$ of dimension $n$. In other words, $H$ is a Lorentzian space. Unlike affine Lie algebras, there are no known general explicit constructions of the hyperbolic ones. It is therefore of interest to try and find more information about their structure and to start with the dimensions of their imaginary root spaces. It is in this connection that Goddard and Thorn's No-Ghost Theorem GodT from theoretical physics was first applied in mathematics by Frenkel [Fre]. This then led to Borcherds' use of this theorem in order to find explicit realizations of generalized 
Kac-Moody algebras whose root lattice is Lorentzian of rank at most 26. Hyperbolic Kac-Moody algebras have recently been extensively studied by Nikulin and Gritsenko [Nik1], Nik2], GriN1], GriN2], GriN3].

2.5. Highest weight modules. Before ending this chapter, we will review some basic facts about an important class of representations of generalized Kac-Moody algebras. Highest weight representations will be needed throughout the rest of the paper. They lead to the denominator formula, which is central to the theory of generalized Kac-Moody algebras because it not only contains the essential information about the corresponding algebra but also provides the link between these algebras and automorphic forms. This link is indeed a very interesting aspect of this area. Furthermore as mentioned in the introduction, the denominator formula is essential in the proof of the Moonshine Conjecture. The construction of vertex algebras is also based on basic highest weight representations of affine Lie algebras.

The highest weight representations of generalized Kac-Moody algebras generalize the finite dimensional representations of the finite dimensional semi-simple Lie algebras. In the case of affine Lie algebras, they were first introduced by Kac Kac2 . The physical motivation in considering this class of representations stems from the requirement that the spectrum of the eigenvalues of the derivation $d$ (see $\S 2$ ) must be bounded above, as for example in string theory the eigenvalues of the operator $-d$ give the mass spectrum.

It should be noted that many representations are neither highest nor lowest weight. Such is the case of the adjoint representation itself when the generalized Kac-Moody algebra is infinite dimensional. Non-highest weight type representations have been little studied, and much remains to be done.

We now define highest weight modules. Let $G$ be a generalized Kac-Moody algebra with Cartan sub-algebra $H$. The $G$-module $V(\Lambda)$ has highest weight $\Lambda \in H^{*}$ if $V(\Lambda)$ is generated by a highest weight vector $v$ satisfying

$$
x v=0, x \in G_{\alpha}, \alpha \in \Delta^{+}, \quad h v=\Lambda(h) v, h \in H .
$$

The weight $\Lambda$ is integrable if $\left(\Lambda, \alpha_{i}\right) \geq 0$ for all simple roots $\alpha_{i}$ of $G$ and $\frac{2\left(\Lambda, \alpha_{i}\right)}{\left(\alpha_{i}, a_{i}\right)} \in \mathbf{Z}$ for all real simple roots $\alpha_{i}$. So if $\Lambda$ is an integrable weight, then for any real simple root $\alpha_{i}$, the action of the corresponding $s l_{2}$ on $V(\Lambda)$ decomposes into finite dimensional $s l_{2}$-representations, or in other words the corresponding generators $e_{i}$ and $f_{i}$ are locally nilpotent on $V(\Lambda)$. When $G$ is a Kac-Moody algebra, the converse also holds since there are no imaginary simple roots. The requirement $\left(\Lambda, \alpha_{i}\right) \geq 0$ for imaginary simple roots is a technical necessity. However note that the highest weight module $V(\Lambda)$ of a generalized Kac-Moody algebra is integrable if and only if it is unitarizable [KacP4], by which we mean that there is a positive definite contravariant bilinear form on $V(\Lambda)$.

For all $\Lambda \in H^{*}$, it can be shown there is a unique (up to isomorphism) $G$-module $M(\Lambda)$ of highest weight $\Lambda$, called the Verma module, such that any $G$-module of highest weight $\Lambda$ is a quotient of $M(\Lambda)$. It follows that for all $\Lambda \in H^{*}$, there is a unique (up to isomorphism) irreducible $G$-module $M(\Lambda)$ of highest weight $\Lambda$, which we will denote $V(\Lambda)$.

\section{Vertex algebras}

In this section we introduce another essential ingredient in the proof of the Moonshine Conjecture. 
3.1. Vertex operators. Roughly speaking a vertex algebra is a vector space with infinitely many formal operators, the vertex operators, which act on it and satisfy certain properties. Generating functions known as (twisted) vertex operators first appeared in the mathematics literature in 1978 in the work of Lepowsky and Wilson LepW] on the basic representations of affine $s l_{2}$, a basic representation of an affine Lie algebra being an integrable highest weight representation $V(\Lambda)$ with $\Lambda(c)=1$, where $c$ is the canonical central element (see $\S 2.4$ ). A construction of the basic representations for all affine Lie algebras using untwisted vertex operators was done by Frenkel and Kac FreK] in 1980. It was also done differently by Segal Seg. Lepowsky and Wilson's work was extended to all affine Lie algebras by Kac, Kazhdan, Lepowsky and Wilson KacKLW] in 1981. Twisted vertex operators were constructed by Kac-Peterson for root lattice $E_{8}$ and automorphisms from Weyl groups KacP5 and, using completely different methods, by Lepowsky for any lattice and any isometry of the lattice [Lep2.

Vertex operators have since played an essential role in the representation theory of affine Lie algebras. In order to construct explicit highest weight representations of affine Lie algebras, it was necessary to use vertex operators which first appeared in theoretical physics, more precisely in the theory of dual resonance, where vertex operators are called quantum fields. They were introduced by Fubini and Veneziano in 1972 Man].

Vertex operators are quantum fields on a vector space $V$, which are, by definition the generating series of endomorphisms of $V$ written

$$
\alpha(z)=\sum_{n \in \mathbf{Z}} \alpha_{(n)} z^{-n-1},
$$

where $\alpha_{(n)}$ are endomorphisms of the space $V$ and for any $v \in V, \alpha_{(n)}(v)=0$ for all $n$ large enough.

3.2. Definition of a vertex algebra. The vertex operator representations of affine Lie algebras inspired a construction of the simply-laced finite dimensional Lie algebras, i.e. of type $A, D, E$, all of whose roots have the same norm [FreK]. The point of this construction is that it yields an explicit Chevalley basis for the Lie algebra. It is also possible to construct certain types of infinite dimensional generalized Kac-Moody algebras starting from lattices and to find in this way an explicit basis for the algebras in question. However the procedure is more complicated and indirect, and one needs to first consider other objects: vertex algebras. Their construction is a generalization of the vertex representation of Kac-Moody algebras all of whose roots have the same length and in particular of affine Lie algebras with this property and is based on Frenkel's application in 1985 [Fre] of the Goddard-Thorn No-Ghost Theorem to the theory of Kac-Moody algebras.

The starting point is an even lattice $L$ with non-degenerate bilinear form (.,.). We remind the reader that a lattice $L$ is a $\mathbf{Z}$-module (in other words a free abelian group) with an integral valued bilinear form. The lattice $L$ is even if for any $\alpha \in L$, $(\alpha, \alpha)$ is even. We need to construct what the physicists call its "Fock space". Since $L$ is even, it can be shown that there is a unique (up to isomorphism) central extension $\hat{L}$ of $L$ by the group $\mathbf{Z}_{2}$ of order 2 :

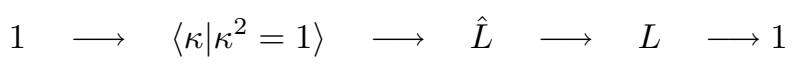


such that $a b a^{-1} b^{-1}=\kappa^{(\bar{a}, \bar{b})}, \quad a, b \in L$, where for $x \in \hat{L}, \bar{x}$ is the image of $x$ in $L$ under the natural projection. Set

$$
H=L \otimes \mathbf{z} \mathbf{R}
$$

and extend the bilinear form to $H$. Consider $H$ as an abelian Lie algebra, and take the extended affine Lie algebra $\tilde{H}$ of $H$ (see $\S 2.4)$ :

$$
\tilde{H}=H \otimes \mathbf{C}\left[t^{-1}, t\right] \oplus \mathbf{C}_{c} \oplus \mathbf{C} d .
$$

For $\alpha \in H$ and $n \in \mathbf{Z}, \alpha(n)$ will denote $\alpha \otimes t^{n}$. The symmetric algebra $S$ of $\oplus_{n<0}\left(H \otimes t^{n}\right)$ is an irreducible natural module for the Heisenberg algebra $[\tilde{H}, \tilde{H}]$. We remind the reader that a Heisenberg algebra is a Lie algebra whose derived subalgebra has dimension 1 and coincides with its centre. For any $x \in S$, writing $x=\beta(-m) y, y \in S$, the action of $\tilde{H}$ on $S$ is defined as follows:

$$
\begin{aligned}
c . x & =x ; \\
\alpha(n) . x & = \begin{cases}\alpha \otimes t^{n} x, & \text { if } n<0 ; \\
0, & \text { if } n=0 ; \\
n \delta_{n, m}(\alpha, \beta) y+\beta(-m) \alpha(n) . y, & \text { if } n>0 .\end{cases} \\
d . \beta(-m) y & =\beta(-m-1) y+\beta(-m) d . y \quad \text { for } m>0 .
\end{aligned}
$$

The Fock space is defined to be the tensor product

$$
V_{L}=S \otimes \mathbf{C}[L],
$$

where $\mathbf{C}[L]$ is the group algebra $\mathbf{C}[\hat{L}]$ quotiented out by $\langle\kappa+1\rangle$. The elements of $\mathbf{C}[\hat{L}]$ are written $e^{\alpha}$, where $\alpha \in L$. The action of $\tilde{H}$ can be extended to the space $V_{L}$ : for any $e^{\beta} \in \mathbf{C}[L]$,

$$
\begin{aligned}
c . e^{\beta} & =0, \\
\alpha(n) . e^{\beta} & =\delta_{n, 0}(\alpha, \beta) e^{\beta}, \\
d . e^{\beta} & =\beta(-1) e^{\beta} .
\end{aligned}
$$

Thus for $n<0, \alpha(n)$ is a "creation operator" and for $n \geq 0, \alpha(n)$ is an "annihilation operator".

Each element of $V_{L}$ has an associated vertex operator. For all $\alpha, \alpha_{1}, \alpha_{2} \ldots \in L$, $Y\left(\alpha t^{-1} \otimes 1, z\right)=\sum_{n \in \mathbf{Z}} \alpha(n) z^{-n-1}$

$Y\left(1 \otimes e^{\alpha}, z\right)=e^{\alpha} z^{\alpha(0)} e^{-\sum_{j<0} \frac{z^{-j}}{j} \alpha(j)} e^{-\sum_{j>0} \frac{z^{-j}}{j} \alpha(j)}$

$Y\left(\left(\alpha_{1} t^{-n_{1}-1}\right)\left(\alpha_{2} t^{-n_{2}-1}\right) \ldots \otimes e^{\alpha}, z\right)$

$$
=: \frac{d}{d z}^{\left(n_{1}\right)} Y\left(\alpha_{1} t^{-1} \otimes 1, z\right) \frac{d}{d z}{ }^{\left(n_{2}\right)} Y\left(\alpha_{2} t^{-1} \otimes 1, z\right) Y\left(1 \otimes e^{\alpha}, z\right):
$$

In the above, the notation :: means that all the creation operators are on the left of all the annihilation operators. This is called the normal ordering [Bor2], [FreLM2], Kac10 and is needed to ensure convergence. For any $v \in V_{L}$, these vertex operators have the following properties [Bor2], FreLM2], Kac10]:

1. $[d, Y(v, z)]=\frac{d}{d z} Y(v, z)$

2. $Y(1, z)=I_{V_{L}},\left.\quad Y(v, z) \cdot 1\right|_{z=0}=v$;

3. $(z-w)^{N}(Y(v, z) Y(w, y)-Y(w, y) Y(v, z))=0 \quad$ for $\quad N>>0$. 
In the above the vector $1 \otimes 1 \in V_{L}$ is written 1 . In equality 3 , the factor $(z-w)^{N}$ cannot be cancelled out. This is often the case for formal series in $z$ and $w$. For example, if $\delta(z-w)=z^{-1} \sum_{n \in \mathbf{Z}}\left(\frac{w}{z}\right)^{n}$, then $(z-w) \delta(z-w)=0$. If the operators $Y(v, z)$ are considered as complex functions, then relation 3 simply says that commutativity holds. However in a strict sense, $Y(v, z) Y(w, y)$ and $Y(w, y) Y(v, z)$ are not always defined in the same domain. So it is better to define vertex operators as formal series.

Based on the above, a vertex algebra is defined to be any vector space $V$ having:

(i) a special vector $1 \in V$; and

(ii) vertex operators $Y(v, z)=\sum_{n \in \mathbf{Z}} v(n) z^{-n-1} \in \operatorname{End}(V)[[z]]$ for every element $v \in V$ depending linearly on $v$ and satisfying properties (1)-(3), where $d \in E n d(V)$ is defined by $d(v)=v_{-2}(1)$ for all $v \in V$.

The above definition given by Dong and Lepowsky [DonL and by Li [Li] (see Kac10] is simpler to state than Borcherds' original one [Bor2], and both are equivalent [DonL], Kac10]. The vertex algebra $V_{L}$ constructed from a lattice $L$ is called a lattice vertex algebra.

Before closing this section, we briefly explain the connection between vertex algebras and highest weight representations of untwisted affine Lie algebras. As mentioned earlier, the original motivation which led to the introduction of vertex operators in mathematics was to explicitly construct such representations.

Let $L$ be a positive definite even lattice and $\Delta$ the set of vectors of norm 2 in the lattice $L$. The elements $e^{\alpha}$ for $\alpha \in \Delta$ give the Chevalley basis of a finite dimensional semisimple Lie algebra $G$ with root system $\Delta$ and Cartan subalgebra $H=L \otimes \mathbf{R}$ :

$$
G=H \oplus\left(\oplus_{\alpha \in \Delta} \mathbf{R} e^{\alpha}\right)
$$

Let $\hat{G}$ be the affinization of $G$ and $\tilde{G}$ its extended affine Lie algebra. From the above construction, the lattice vertex algebra $V_{L}$ can be shown to be a representation of $\tilde{G}$. The action of the elements $c, d$ and $\alpha(n)$ of $\tilde{G}$ for $\alpha \in H$ is given by the representation of the extended affinization of $H$ on $V_{L}$, and the action of $e^{\alpha} \otimes$ $t^{n}$ for $\alpha \in \Delta$ is given by the coefficient of $z^{-n-1}$ in the operator $Y\left(e^{\alpha}, z\right)$. Let $Q=\mathbf{Z} \Delta$ be the lattice spanned by $\Delta$. As a $\tilde{G}$-representation, $V_{L}$ is the direct sum of the fundamental representations of $\hat{G}$ corresponding to the orbits of $Q$ in $L$. Furthermore the vertex algebra $V_{Q}$ is the distinguished fundamental basic $\tilde{G}$ representation, which plays an important role in theory of affine algebras [FreK], FreLM2. It is known as the Frenkel-Kac construction. Each simply-laced affine Lie algebra $\hat{G}$ has a twisted vertex operator representation for each conjugacy class of the Weyl group of $G$, and the Frenkel-Kac construction corresponds to the trivial conjugacy class [KacP5], Lep2.

3.3. The No-Ghost Theorem and generalized Kac-Moody algebras. We will now explain how for certain even non-degenerate lattices $L$, one gets a generalized Kac-Moody algebra from the lattice vertex algebra $V_{L}$. We keep the notation of section 3.2 .

We first need to show how the Virasoro algebra acts on the space $V_{L}$. We suppose in the rest of this section that $L$ is an even non-degenerate lattice. The lattice vertex algebra $V_{L}$ is a vertex algebra with a special property. It contains a vector which 
can be defined as follows:

$$
\nu=\frac{1}{2} \sum_{i} \alpha_{i}(-1) \alpha_{1}^{\prime}(-1)
$$

where the vectors $\left(\alpha_{i}\right)$ run through a basis of $L$ and $\left(\alpha_{1}^{\prime}\right)$ through a dual basis in $H$. This vector $\nu \in V_{L}$ is a "conformal vector"; i.e. its corresponding vertex operator can be written

$$
Y(\nu, z)=\sum_{n \in \mathbf{Z}} L_{n} z^{-n-2},
$$

where the operators $L_{n}, n \in \mathbf{Z}$, together with a central element $c$ acting as multiplication by the rank of the lattice $L$ on the space $V_{L}$, generate a Virasoro algebra FreK]. In particular, $L_{-1}=d$ and $L_{0}$ is diagonalizable on $V_{L}$ : for every $\alpha \in L$ and $h \in H$,

$$
L_{0} e^{\alpha}=\frac{1}{2}(\alpha, \alpha) e^{\alpha} \quad \text { and } \quad L_{0}(h(-n) v)=n h(-n) v+h(-n) L_{0} v, \quad v \in V_{L} .
$$

The important point is that we have an action of the Virasoro algebra on $V_{L}$. We remind the reader that the Virasoro algebra is the unique (up to isomorphism) central extension by a 1 dimensional centre of the Lie algebra of all derivations of the algebra of Laurent polynomials $\mathbf{C}\left[t, t^{-1}\right]$. It is generated by a central element $c$ and elements $L_{n}, n \in \mathbf{Z}$, satisfying

$$
\left[L_{m}, L_{n}\right]=(m-n) L_{m+n}+\frac{1}{12}\left(m^{3}-m\right) \delta_{m+n, 0} c .
$$

Lattice vertex algebras $V_{L}$ have conformal vectors because the lattice $L$ is assumed to be non-degenerate. In general a vertex algebra does not contain such a vector.

Note that vertex operator algebras were later defined by Frenkel, Lepowsky and Meurman [FreLM2] to reflect some crucial features of the moonshine module. They are vertex algebras with a conformal vector but with the added condition that all the eigenspaces of $L_{0}$ on $V$ are finite dimensional. The converse is false since lattice vertex algebras are evidently not vertex operator algebras when the non-degenerate even lattice $L$ contains roots of non-positive norms. In this case, the above definition of the operator $L_{0}$ implies that for any $\alpha \in L$ and $h \in H$,

$$
L_{0}\left(h\left(1-\frac{1}{2}(\alpha, \alpha)\right) \otimes e^{\alpha}\right)=h\left(1-\frac{1}{2}(\alpha, \alpha)\right) \otimes e^{\alpha}
$$

whenever $(\alpha, \alpha) \leq 0$.

Before proceeding further, we need to mention that there is a natural nondegenerate bilinear form on $V_{L}$ satisfying

(i) $\quad\left(e^{\alpha}, e^{\beta}\right)=\left\{\begin{array}{ll}1, & \text { if } \alpha=\beta ; \\ 0, & \text { otherwise; }\end{array}\right.$ and

(ii) the adjoint of the operator $\alpha(-n)$ for any $\alpha \in H$ is $\alpha(n)$.

The aim is to construct a generalized Kac-Moody algebra from $V_{L}$.

For any vertex algebra $V$, the quotient space $V / d V$, where $d V$ is the subspace generated by the action of the derivation $d$ on $V$, can be given the structure of a Lie algebra by defining the bracket as follows:

$$
[v, w]=v_{0}(w), \quad v, w \in V,
$$

where $v_{0}$ is the coefficient of $z^{-1}$ in the operator $Y(v, z)$. 
However Goddard-Thorn GodT] and Frenkel [Fre] showed that the space we should consider is not the whole of the lattice vertex algebra $V_{L}$ but a special subspace $V_{1}$ of $V_{L}$, where for all $n \in \mathbf{N}$

$$
V_{n}=\left\{v \in V \mid L_{0} v=n v, L_{i} v=0, \forall i>0\right\} .
$$

Since $d V \cap V_{1}=d V_{0}$, it follows from the above that $V_{1} / d V_{0}$ has the structure of a Lie algebra.

If $L$ contains the root lattice of a Kac-Moody algebra $G$ all of whose real roots have the same length, it can then be shown that there is a Lie subalgebra of the Lie algebra $V_{1} / d V_{0}$ isomorphic to the derived Lie algebra $G^{\prime}$. It is generated by the elements

$$
e_{i}=e^{\alpha_{i}}, f_{i}=f^{\alpha_{i}}, h_{i}=\alpha_{i}(-1) .
$$

This gives upper bounds for the multiplicities of the roots of $G$. Frenkel's insight was to apply the Goddard-Thorn No-Ghost Theorem [GodT] of 1972 from the theory of dual resonance in theoretical physics to this context in order to find better upper bounds when $G$ is a hyperbolic Lie algebra (see $\$ 2.4$ ) of rank at most 26 [Fre].

Generalized Kac-Moody algebras were unknown at this time, and it is in 1992 that using his characterization theorem (see §2.3) Borcherds showed that a quotient of $V_{1} / d V_{0}$ is a generalized Kac-Moody algebra Bor5 whose Lie subalgebra generated by the real simple roots is the hyperbolic Lie algebra $G$ studied by Frenkel.

We give a brief outline of the No-Ghost Theorem and of its proof GodT], [Fre], Bor7, Jur. Let $L$ be an even Lorentzian lattice and $G$ the corresponding hyperbolic Lie algebra. As before, $H=L \otimes \mathbf{C}$ and the construction of $\S 3.2$. shows that the lattice vertex algebra $V_{L}=S \otimes \mathbf{C}[L]$ is a representation of $G$ since all the real roots of $G$ have the same length. The space $V_{L}$ has an $L$-grading given by

$$
\operatorname{deg}\left(s \otimes e^{\alpha}\right)=\alpha \quad \text { for } \quad \alpha \in L, \quad s \in S .
$$

For $\alpha \in L$, set

$$
V_{L}^{\alpha}=\left\{v \in V_{L} \mid \operatorname{deg}(v)=\alpha\right\} \quad \text { and } \quad V_{1}^{\alpha}=V_{1} \cap V_{L}^{\alpha} .
$$

Since $L_{0}\left(h_{1}\left(n_{1}\right) \ldots h_{r}\left(n_{r}\right) \otimes 1\right)=-\left(n_{1}+\ldots n_{r}\right) h_{1}\left(n_{1}\right) \ldots h_{r}\left(n_{r}\right) \otimes 1$ for all $h_{1}, \ldots, h_{r} \in H$, $n_{1}, \ldots, n_{r}<0$, and $L_{n}(h(-1) \otimes 1)=0$ for all $n \geq 1, h \in H$,

$$
V_{1}^{0}=\{h(-1) \otimes 1 \mid h \in H\} .
$$

Furthermore

$$
(h(-1), h(-1))=(h, h),
$$

so that $V_{1}^{0}$ contains negative norm vectors as $L$ is Lorentzian.

The No-Ghost Theorem GodT states the absence of ghosts (i.e. of vectors of negative norm) in $V_{1}^{\alpha}$ when $\alpha \neq 0$ in the Veneziano model if and only if the space-time dimension is less than the critical dimension 26. This result is not at all obvious.

Fix a non-zero root $\alpha \in L$. Since $L$ is Lorentzian, there is an isotropic vector $\beta \in L$ (i.e. $(\beta, \beta)=0$ ) which is not perpendicular to $\alpha$. Set

$$
P=\left\{v \in V^{\alpha} \mid L_{n}(v)=0, n>0\right\},
$$

and let

$$
T=\left\{v \in V^{\alpha} \mid L_{n} v=0=\beta(n) v, \forall n \geq 1\right\}
$$


be the transverse subspace of $P$. Let $N$ be the null space of $\left.(.,)\right|_{.V_{1}}$. Then $V_{1}^{\alpha}=$ $\left\{v \in P \mid L_{0}(v)=v\right\}$. The first step is to show that

$$
V_{1}^{\alpha}=T_{1} \oplus N^{\alpha},
$$

where $T_{1}=T \cap V_{1}$ and $N^{\alpha}=N \cap V^{\alpha}$.

As $(\alpha, \beta) \neq 0$, it can be shown that the bilinear form is non-degenerate on the subspace $T$ and that the space $V^{\alpha}$ is generated by elements of the form

$$
L_{s_{1}} L_{s_{2}} \ldots \beta\left(r_{1}\right) \beta\left(r_{2}\right) \ldots(v),
$$

where $0>s_{1} \geq s_{2} \ldots, 0>r_{1} \geq r_{2} \ldots$, and $v \in T$.

Set $C$ to be the subspace of $V^{\alpha}$ generated by elements of the above type with $s_{1} \neq 0$ and $K$ the subspace generated by the action of the operators $\beta(n)$ on $T$, for all $n \in \mathbf{Z}$. Let $v \in V_{1}^{\alpha}$. There exists $v_{1} \in K$ and $v_{2} \in C$ such that $v=v_{1}+v_{2}$. Since $\left[L_{m}, \beta(n)\right]=-n \beta(n+m)$, for all $m>0, L_{m}\left(v_{1}\right)$ is contained in $K$. Since $\operatorname{dim} L=26$, using the above formula for commutators of elements in the Virasoro algebra, one can prove that the spaces $L_{1}\left(V^{\alpha}\right)$ and $\left(L_{2}+\frac{3}{2} L_{1}\right)\left(V^{\alpha}\right)$ are contained in $C$. Hence as $L_{1}$ and $L_{2}+\frac{3}{2} L_{1}$ generate $\left\langle L_{m}\right| m>0>$, for all $m>0, L_{m}\left(v_{2}\right)$ is in $C$. By definition, $L_{m} v=0$ for $m>0, K \cap C=0$, and $(C, P)=0$. It follows that $v_{1} \in K \cap P$ and $v_{2} \in C \cap P$. Since $(\beta, \beta)=0, K \cap P=T$. As the bilinear form is non-degenerate on $T$, we can therefore conclude that (1) holds and so there are no negative norm vectors in $V_{1}^{\alpha}$ if $\alpha \neq 0$.

The second step is to check that the Lie algebra $V_{1} / d V_{0}$ has a quotient which satisfies the conditions of Borcherds' characterization theorem. Since $L$ is Lorentzian, $\operatorname{dim}\left\{h \in H_{\mathbf{R}} \mid(h, h)<0\right\}=1$. As the form is non-degenerate on $T$, this implies that the form is in fact positive definite on $T$. The involution $\omega$ acting on the space $V_{L}$ by

$$
\omega\left(e^{\alpha}\right)=-(-1)^{(\alpha, \alpha) / 2}\left(e^{\alpha}\right)^{-1}, \quad \omega(h(n))=-h(-n), h \in H, n \in \mathbf{Z}
$$

induces an involution on the Lie algebra $V_{1} / d V_{0}$. The form (.,.) is contravariant with respect to $\omega$. Therefore $N$ is an ideal in $V_{1}$. Hence $V_{1} / N$ is a Lie algebra, which we will call $F$. Also $d V_{0} \leq N$ as $\left(d V_{0}, V_{1}\right)=0$. As $L$ is Lorentzian, we can choose a root $\gamma$ in $L$ of negative norm not orthogonal to any norm 2 root of $L$. The inner product with $\gamma$ gives a $\mathbf{Z}$-grading of $F$. With this grading, the bilinear form induced on $F$ by the form on $V_{L}$ and the involution satisfy the condition of Borcherds' Theorem since the No-Ghost Theorem implies this form is positive definite almost everywhere on $F$. Therefore $F$ is a generalized Kac-Moody algebra.

If the lattice $L$ is also assumed to be unimodular (i.e. $L$ is self dual: $L=L^{*}$ ), then the generalized Kac-Moody algebra $F$ is called the Fake Monster Lie algebra, and it was the first explicit example of a generalized Kac-Moody algebra which is not a Kac-Moody algebra. It was constructed by Borcherds in 1990 [Bor5]. In [Bor5], Borcherds calculates the root multiplicities of the real roots of this Lie algebra all having norm 2. The hyperbolic Lie algebra $G$ of rank 26 studied by Frenkel is a Lie subalgebra of the generalized Kac-Moody algebra $F$, and the real root spaces of $G$ and $F$ coincide. In [Bor5], Borcherds also calculates the root multiplicities of the Fake Monster Lie algebra. They are equal to the upper bounds for these multiplicities given by Frenkel: For any root $\alpha$ of the Fake Monster Lie algebra

$$
\operatorname{dim} F_{\alpha}=p_{24}(1-(\alpha, \alpha) / 24),
$$


where $p_{24}(n)$ is the number of partitions of $n$ into parts of 24 colours. Note that this implies that

$$
\operatorname{dim} G_{\alpha} \leq p_{24}(1-(\alpha, \alpha) / 24),
$$

where $G$ is the hyperbolic Lie algebra of rank 26, and thus we get Frenkel's earlier result Fre when the lattice $L$ is unimodular.

Before Borcherds constructed the class of generalized Kac-Moody algebras, it had been thought that the Kac-Moody algebra $G$ generated by the simple roots of norm 2 contained in the Fake Monster Lie algebra was the Lie algebra that would explain the Moonshine Conjecture. This is because the unimodular even Lorentzian lattice of dimension 26 is closely connected to the Leech lattice ConS.

After constructing the Fake Monster Lie algebra, Borcherds at first thought this to be the suitable Lie algebra. It was therefore originally called the Monster Lie algebra, and its name was later changed.

Note that the above result can be easily extended to even Lorentzian lattices of any rank less than 26 and that the real roots of the generalized Kac-Moody algebras thus constructed all have norm 2 .

The interesting hyperbolic Kac-Moody algebras are those which like $G$ are contained in a generalized Kac-Moody algebra or superalgebra in such a way that both have the same real root spaces. Based on Borcherds' work, the theory of this type of hyperbolic Lie algebras has been developed in depth by Nikulin and Gritsenko. For a given rank $r \geq 3$, there are only finitely many root lattices possible for such hyperbolic Lie algebras [Nik2].

3.4. Quantum vertex algebras. We make a small digression in this section. The definition of a vertex algebra suggests that it is some kind of ring acted on by a group Bor2. This becomes clear in the most simple case, i.e. that of a holomorphic vertex algebra. A vertex algebra is holomorphic if for all $a \in V, a_{(n)}=0$ for all $n \geq 0$, so that $Y(a, z)=\sum_{n \in \mathbf{Z}_{+}} a_{-n-1} z^{n}$. Hence the vertex operators $Y(a, z)$ are power series in $z$ and the defining axiom 3 becomes commutativity. One can define an algebra multiplication by

$$
a b=a_{-1} b .
$$

The linear map $d$ of axiom 1 can then be shown to be a derivation of $V$. The vertex operator $Y(a, z)$ can be expressed in terms of this product and the derivation $d$ :

$$
Y(a, z) b=\left(e^{z d} a\right) b,
$$

where $e^{z d}$ is the formal power series for the exponential. It can be shown that holomorphic vertex algebras are the same as commutative associative algebras with a unit element and a derivation $d$ Kac10]. This is easier to see if we use a different notation. Put

$$
a^{z} b:=Y(a, z) b .
$$

So the aim is to try and define a category whose commutative rings are vertex algebras. In [Bor12, Borcherds constructs additive symmetric tensor categories whose singular commutative rings are vertex algebras. This definition generalizes the above original definition of an ordinary vertex algebra, which corresponds to a particular category and allows a construction of quantum vertex algebras as the singular braided rings of the category. Though this definition is in some sense more abstract than the first one, it has the advantage of making the notion of vertex 
algebras appear natural within a mathematical context. The braided rings in the particular category corresponding to the ordinary vertex algebras correspond to the quantum vertex algebras defined earlier by others [EtinK], [FreJ]. These new constructions lead to many open problems. Among others one should verify if there is any link between some quantum vertex algebras and quantum generalized KacMoody algebras (as defined by Kang in [Kan3]) similar to the above one between ordinary vertex algebras and generalized Kac-Moody algebras, and in particular what happens in the case of the Monster Lie algebra.

3.5. The moonshine module. We want to find representations $V(n)$ of the Monster group $M$ of dimension $c(n)$ (see $\S 1$ ), or equivalently we want to construct a graded $M$-module $V=\sum_{n \in \mathbf{Z}} V(n)$ of dimension $J(q)$.

An essential feature of vertex algebras is their link with modular functions. As we saw in $\S 3.2$, vertex operators first appeared in the context of the representation theory of affine Lie algebras. There is a deep connection between affine Lie algebras and modular functions, which was revealed through works of Feingold-Lepowsky FeinL, Kac [Kac2, Kac6 and Kac-Peterson [KacP1], KacP3. The graded dimensions of the basic representations of simply-laced affine Lie algebras are given by modular forms.

For example, in the case of affine $\hat{s}_{2}$, the are two basic representations (see $\S 3.2$ ) are isomorphic to each other. Therefore their graded dimensions which have been calculated in different ways are equal. This leads to the "classical" Gauss formula for the Dedekind $\eta$-function:

$$
\frac{\eta(q)}{\eta\left(q^{1 / 2}\right)}=\frac{\sum_{n \in \mathbf{Z}} q^{\left(n+\frac{1}{4}\right)^{2}}}{\eta(q)}
$$

We remind the reader that

$$
\eta(q)=q^{\frac{1}{24}} \prod_{n=1}^{\infty}\left(1-q^{n}\right) .
$$

Relevant observations by McKay, Garland [Gar, Kac [Kac6], [Kac7] and Lepowsky Lep1] added evidence to the existence of the moonshine module. Before describing the module, we will give a brief description of these other works.

It has been known since the work of Dedekind that the modular invariant $j$ can be written as follows:

$$
j(\tau)=\frac{\theta_{L}(\tau)}{\eta(\tau)^{24}}
$$

where $\theta_{L}(q)=\left(\sum_{\alpha \in \Gamma_{8}} q^{(\alpha, \alpha) / 2}\right)^{3}$ for $q=e^{2 \pi i \tau}$ and $\Gamma_{8}$ is the root lattice of $E_{8}$. Now the graded dimension of the untwisted vertex operator representation $V_{\Gamma_{8}}$ of affine $\hat{E}_{8}$ is $j(q)^{1 / 3}$. Therefore when $L=\Gamma_{8} \oplus \Gamma_{8} \oplus \Gamma_{8}$, the graded dimension of $V_{L}$ is $j(q)=J(q)+744$, which is almost the graded dimension of the module $V$ we want, except that it has a constant term.

The vertex algebra construction described in $\S 3.2$ can be applied to the Leech lattice $\Lambda$. This is the unique 24 dimensional even unimodular positive definite lattice having no vectors of norm 2 (see $\S 7$ ). The graded dimension of $V_{\Lambda}$ is $J(\tau)+24$. Again there is a constant term discrepancy. In Kac8, Kac attempted to construct a module over the centralizer of an involution in the Monster group by changing 
the previous vertex algebra $V_{L}$, and he thought that this module could be extended to the whole Monster group by making use of vertex operators.

Then in 1984, Frenkel, Lepowsky and Meurman [FreLM1 successfully constructed the moonshine module $V$. It can be written as a direct sum of two subspaces:

$$
V=V_{\Lambda}^{+} \oplus\left(V_{\Lambda}^{\prime}\right)^{+}
$$

The vector space $V_{\Lambda}^{+}$is a subspace of the space $V_{\Lambda}$. For any non-degenerate lattice $L$, there are twisted vertex operator constructions corresponding to the conjugacy classes of the automorphism group of $L$ which generalize the affine case [FreLM1, KacP5. Here, $V_{L}^{\prime}$ corresponds to the conjugacy class of -1 and $\left(V_{\Lambda}^{\prime}\right)^{+}$is a subspace of $V_{\Lambda}^{\prime}$. Frenkel, Lepowsky and Meurman showed $V$ has graded dimension $J(q)$ and the structure of a vertex operator algebra. They also proved the important result that the group of vertex algebra automorphisms of $V$ preserving its grading is the Monster group. The moonshine module $V$ contains the Griess algebra $\mathcal{B}$ of dimension 196884. More precisely, the Virasoro operator acts on $V$, and $\mathcal{B}$ is the 2 -eigenspace of the operator $L_{0}$. The algebra $\mathcal{B}$ was constructed by Griess in 1981 Gri], and he gave an explicit construction of the Monster group as a subgroup of the automorphism group of $\mathcal{B}$ by finding adequate symmetries of this algebra. In fact the Monster group is the full group of automorphisms of $\mathcal{B}$ [Tits].

The reason why the traces $T_{g}$ of the action of the element $g$ in the Monster group $M$ on the moonshine module $V$ cannot be computed directly for most elements $g \in G$ is a consequence of the structure of $V$. The group $M$ contains a subgroup $C$ Gri] which is the centralizer of a certain involution of $M$. The subspaces $V_{\Lambda}^{+}$ and $\left(V_{\Lambda}^{\prime}\right)^{+}$are the eigenspaces of this involution. When $g \in C$, the trace $T g$ can therefore be computed [FreLM1, [FreLM2]. When $g$ does not commute with the involution fixing the above two subspaces, it is too complex a calculation. Therefore Borcherds' very elegant proof is needed. In it he applies the theory of Vertex algebras and the No-Ghost Theorem to the moonshine module $V$ in a very original way to get an explicit realization of a generalized Kac-Moody algebra which is acted on by the Monster group, namely the Monster Lie algebra, so that he can then use the representation theory of these algebras to proceed further.

The important aspects of this moonshine module $V$ for our purposes are as follows [FreLM1], [FreLM2]:

1) The $M$-module $V$ has the structure of a vertex algebra.

2) The module $V$ contains a conformal vector of central charge 24 , and so the Virasoro algebra $\left\langle L_{i}, 1 \mid i \in \mathbf{Z}\right\rangle$ acts on $V$ in such a way that

$$
\left[L_{i}, L_{j}\right]=(i-j) L_{i+j}+\left(i^{3}-i\right) \delta_{i,-j} \frac{24}{12} .
$$

The eigenspaces of the operator $L_{0}$ on $V$ are precisely the head representations of the Monster group $M$ :

$$
V(n)=\left\{v \in V \mid L_{0} v=(n+1) v\right\} .
$$

Since $V(n)=0$ for $n<-1$, it follows that $V$ is the direct sum of the eigenspaces corresponding to non-negative integral eigenvalues of $L_{0}$ :

$$
V=\oplus_{n=-1}^{\infty} V(n) .
$$

Note that since for all $n \in \mathbf{Z}, V(n)$ is finite dimensional, the moonshine module $V$ is not only a vertex algebra but also a vertex operator algebra (see $\S 3.3$ ). 
3) There is a positive definite bilinear form (.,.) on $V$.

3.6. The Monster Lie algebra. The construction of the Monster Lie algebra, the most well known generalized Kac-Moody algebra which is not a Kac-Moody algebra and which is central to the proof of the conjecture, brings together everything we have seen so far. In particular, Borcherds had the original insight to adapt the No-Ghost Theorem to the context of the moonshine module $V$, not only to give an explicit realization of the Monster Lie algebra and show that it is a generalized Kac-Moody algebra $\mathcal{M}$, but also to prove that the action of Monster group $M$ on $V$ induces an action on $\mathcal{M}$ such that the root spaces are isomorphic to the head representations.

As written above, the moonshine module $V$ is a vertex algebra. In order to apply the results of $\S 3.3$ to construct a generalized Kac-Moody algebra, we need a vertex algebra graded by a unimodular even Lorentzian lattice of rank at most 26 . The smallest possible rank of a unimodular even Lorentzian lattice is 2, and it can be easily shown that tensor products of vertex algebras are also vertex algebras. The Virasoro algebra acts on the module $V$ with central charge 24 (see §3.5). This suggests that we consider the tensor product of the vertex algebra $V$ with the lattice vertex algebra $V_{I I_{1,1}}$, where $I I_{1,1}$ is the unique even unimodular Lorentzian lattice of rank 2:

$$
B=V \otimes V_{I I_{1,1}}
$$

The bilinear form on $I I_{1,1}$ is given by the matrix $\left(\begin{array}{cc}0 & -1 \\ -1 & 0\end{array}\right)$. The $I I_{1,1}$-grading extends naturally to $B$ by defining $\operatorname{deg}(v \otimes w)=\operatorname{deg}(w)$ for $v \in V, w \in V_{I I_{1,1}}$. Since the Monster group $M$ acts on the space $V$, its action can be extended to the vertex algebra $B$ by making $M$ act trivially on $V_{I I_{1,1}}$.

Set $P_{1}=\left\{v \in B \mid L_{n} v=0, n>0, L_{0} v=v\right\}$, and define

$$
\mathcal{M}=\frac{P_{1}}{\left.\operatorname{Rad}(., .)\right|_{V_{1}}} .
$$

The quotient space $\mathcal{M}$ is a $I I_{1,1}$-graded Lie algebra (see $\S 3.3$ ) called the Monster Lie algebra. We take the $\mathbf{Z}$-gradation defined by: $\operatorname{deg}(v)=2 m+n$ for all $v \in \mathcal{M}_{(m, n)}$, $(m, n) \in \mathbf{Z}^{2}$. Since the lattice $I I_{1,1}$ is unimodular, even and Lorentzian, and the Virasoro algebra acts on $B$ with central charge 26 , we can conclude that $\mathcal{M}$ is a generalized Kac-Moody algebra graded by the lattice $I I_{1,1}$ :

$$
\mathcal{M}=\oplus_{(m, n) \in \mathbf{Z}^{2}} \mathcal{M}_{(m, n)} .
$$

It remains to show that the Monster group $M$ acts on the Lie algebra $\mathcal{M}$ in such a way that the root space $\mathcal{M}_{(m, n)}$ is isomorphic to the $M$-module $V(m n)$. This is also a consequence of the No-Ghost Theorem and of Frenkel, Lepowsky and Meurman's result. Since the Monster group $M$ acts on the module $V$, we can extend its action on the vertex algebra $V \otimes V_{L}$ by making $M$ act trivially on $V_{L}$. It can be shown that as a $M$-module, $V \otimes e^{\alpha}$ is isomorphic to the transverse subspace $T$ (its definition is adapted in the obvious way from the one given in $\S 3.3$ in the case of the Lorentzian lattice of rank 26). By the No-Ghost Theorem, when the root $\alpha$ is non-zero, $\mathcal{M}_{\alpha}$ is isomorphic to the subspace $T_{1}$ of $T$ of elements fixed by the operator $L_{0}$ (see $\S 3.3$ ). By what precedes, $\mathcal{M}_{\alpha}$ is therefore isomorphic to 
$\left\{v \otimes e^{\alpha} \mid v \in V, L_{0}\left(v \otimes e^{\alpha}\right)=v \otimes e^{\alpha}\right\}$. From the action of $L_{0}$ on $V_{L}$, it can be deduced easily that

$$
\mathcal{M}_{\alpha} \cong V_{-\frac{1}{2}(\alpha, \alpha)}=\left\{v \in V \mid L_{0} v=\left(1-\frac{1}{2}(\alpha, \alpha)\right) v\right\} .
$$

In other words, as a $M$-module, for $\alpha=(m, n)$ with $(m, n) \neq(0,0)$,

$$
\mathcal{M}_{(m, n)} \cong V(m n)
$$

since $(m, n) .(m, n)=-2 m n$. Furthermore, setting $H=L \otimes \mathbf{z} \mathbf{R}$,

$$
B_{0}=V(-1) \otimes\{h(-1) \mid h \in H\}
$$

is isomorphic to $\mathbf{R}^{2}$ since $V(-1)$ is the trivial $M$-module of dimension 1 and $H \cong \mathbf{R}^{2}$. Therefore as an $M$-module

$$
\mathcal{M} \cong \mathbf{R}^{2} \oplus\left(\oplus_{(m, n) \in \mathbf{Z}^{2}} V(m n)\right) .
$$

(Note that $M$ acts trivially on $\mathbf{R}^{2}$.)

\section{Denominator Formulas And AUtomorphic Forms}

We have now constructed the Monster Lie algebra $\mathcal{M}$. We know exactly what its Cartan subalgebra $H$ is, what the bilinear form on it is, and the dimensions of all its root spaces. In order to proceed further we need to identify which among the roots are simple; that is, we need to find the generators of $\mathcal{M}$. Otherwise we will not be able to use the homology of generalized Kac-Moody algebras which will lead us to a formula which relates the traces of different elements of the Monster group on the various $M$-modules $V(n)$.

Furthermore the next step in the proof of the Moonshine Conjecture illustrates how the theory of generalized Kac-Moody algebras can be a framework for interpreting some identities involving automorphic forms. We have to compute the denominator formula of the Monster Lie algebra $\mathcal{M}$ to find the simple roots of $\mathcal{M}$. So we now explain what the denominator formula of a generalized Kac-Moody algebra is.

4.1. The denominator formula. Let us fix some notation:

$G$ will denote a generalized Kac-Moody algebra, $H$ a Cartan sub-algebra, and $H^{*}$ the dual space of $H$.

The roots will be considered to be elements in $H^{*}$ (see $\S 2.2$ ), and the simple roots will be written $\alpha_{i}, i \in I$.

$\Delta^{+}$will denote the set of positive roots of $G$, and for all $\alpha \in \Delta^{+}, \operatorname{mult}(\alpha)$ will denote the dimension of the root space $G_{\alpha}$.

$W$ will denote the Weyl group of $G$, i.e. $W=\left\langle r_{i} \mid i \in I,\left(\alpha_{i}, \alpha_{i}\right)>0\right\rangle$, where $r_{i}: H^{*} \rightarrow H^{*}$ is defined by $r_{i}(\alpha)=\alpha-\frac{2\left(\alpha, \alpha_{i}\right)}{\left(\alpha_{i}, \alpha_{i}\right)} \alpha_{i}$. The length of $w \in W, l(w)$, is the minimal number of generators $r_{i}$ needed to write $w$.

$\rho \in H^{*}$ will denote the Weyl vector, which we assume exists, i.e. $\rho\left(\alpha_{i}\right)=$ $\frac{1}{2}\left(\alpha_{i}, \alpha_{i}\right)$ for all $i \in I$.

Let $V(\Lambda)$ be a $G$-module with highest weight $\Lambda \in H^{*}$ and highest weight vector $v_{\Lambda}$ (see $\S 2.5$ for definition). The Cartan sub-algebra $H$ acts semi-simply on $V(\Lambda)$. The eigenspaces

$$
V_{\lambda}=\{v \in V(\Lambda) \mid h v=\lambda(h) v, h \in H\}, \lambda \in H^{*}
$$


are called weight spaces. So

$$
V(\Lambda)=\oplus_{\lambda \in H} V_{\lambda} .
$$

The Poincaré-Birkhoff-Witt Theorem implies that all the weight spaces are finite dimensional.

The first natural question is to compute the dimensions of the weight spaces, in other words to find the character of $V(\Lambda)$ defined as the following formal sum:

$$
\chi(V(\Lambda))=\sum_{\lambda \in H^{*}} \operatorname{dim} V_{\lambda} e(\lambda),
$$

where $e(\lambda)$ is the formal exponential. These sums belong to the set of formal series $\sum_{\lambda \in H^{*}} x_{\lambda} e(\lambda)$, where the coefficients $x_{\lambda}$ are non-trivial only inside a finite union of sets like $\left\{\alpha \in H^{*} \mid \alpha \leq \beta\right\}$. The elements $e(\lambda)$ of this set behave as the usual exponential with respect to addition and multiplication.

Let $V(\Lambda)$ be the unique (up to isomorphism) irreducible $G$-module of highest weight $\Lambda$ (see $\S 2.5$ ). When $\Lambda$ is an integrable weight (see $\S 2.5$ ), the character formula gives an expression for the character of $V(\Lambda)$ :

$$
\chi(V(\Lambda))=\frac{\sum_{w \in W}(-1)^{l(w)} w\left(e^{\rho} \sum_{\alpha} \epsilon(\alpha) e^{\alpha+\Lambda}\right)}{e^{\rho} \prod_{\alpha \in \Delta^{+}}\left(1-e^{-\alpha}\right)^{\operatorname{mult} \alpha}},
$$

where

$$
\epsilon(\alpha)= \begin{cases}(-1)^{n} & \text { if } \alpha \text { is the sum of } n \text { mutually orthogonal simple imaginary } \\ 0 & \text { roots } \alpha_{i} ; \\ \text { otherwise. }\end{cases}
$$

Note that we have assumed the roots to be elements of $H^{*}$ rather than in the root lattice or the extended Cartan subalgebra. Imaginary roots may have restricted simple root multiplicities $>1$ (see $\S 2.2$ ). Therefore to calculate $\epsilon(\alpha)$, it is necessary to consider the set of simple roots as a set containing $s$ copies of the root $\beta$ if its restricted simple root multiplicity equals $s$.

The denominator formula follows from the character formula for the trivial representation of dimension 1 :

$$
e^{-\rho} \sum_{w \in W}(-1)^{l(w)} w\left(e^{\rho} \sum_{\alpha} \epsilon(\alpha) e^{\alpha}\right)=\prod_{\alpha \in \Delta^{+}}\left(1-e^{-\alpha}\right)^{\mathrm{mult} \alpha} .
$$

The denominator formula was proved by Borcherds in the general setup of generalized Kac-Moody algebras [Bor4]. It essentially characterizes the algebra $G$ as it is equivalent to knowing what the roots are and what the simple roots are since the Weyl group is generated by simple real root reflections. The main characteristic of the denominator formula is that on one side you have a sum and on the other a product. This is why it gives in many cases interesting identities, or more precisely product expansions for automorphic forms. If $G$ is a Kac-Moody algebra, then $G$ does not contain any imaginary simple roots. Hence in this case the denominator formula reduces to

$$
\sum_{w \in W}(-1)^{l(w)} w\left(e^{\rho}\right)=e^{\rho} \prod_{\alpha \in \Delta^{+}}\left(1-e^{-\alpha}\right)^{\text {mult } \alpha} .
$$

This was proved by Kac in 1974 [Kac2]. So in the more general case of generalized Kac-Moody algebras, there is simply an added factor on the left hand side depending on mutually commuting imaginary simple roots. When $G$ is finite dimensional, all 
roots are real, so all root multiplicities equal 1. The denominator formula in this case is the classical Weyl formula.

4.2. Affine Lie algebras and the Macdonald identities. The only infinite dimensional Kac-Moody algebras for which we have explicit formulations for their denominator formula are the affine Lie algebras. In this section we illustrate how their denominator formula is nothing but the famous identities Macdonald found in 1972 as a generalization of the Weyl formula to what he called affine systems [Mac. However he did not account for some extra factors appearing in these identities. Shortly afterward Kac proved the denominator formula for Kac-Moody algebras Kac2 and found that the Macdonald identities were the denominator formulas for the affine Lie algebras.

We give an example. Consider the simplest affine Lie algebra, $s \hat{l}_{2}$, otherwise known as $A_{1}^{(1)}$. Its Cartan matrix is $\left(\begin{array}{cc}2 & -2 \\ -2 & 2\end{array}\right)$, and so it is obtained by adding one simple root to the 3 -dimensional Lie algebra $s l_{2}$. Let $\alpha_{1}$ be the simple root of $s l_{2}$, and $\alpha_{0}, \alpha_{1}$ the simple roots of $s \hat{l}_{2}$. To find the right hand side of (2), we need to know the set $\Delta^{+}$of positive roots. The Cartan matrix implies that

$$
\Delta^{+}=\left\{n \alpha_{0}+(n-1) \alpha_{1}, n\left(\alpha_{0}+\alpha_{1}\right),(n-1) \alpha_{0}+n \alpha_{1}, n \geq 1\right\} .
$$

Since the roots $n \alpha_{0}+(n-1) \alpha_{1}$ and $(n-1) \alpha_{0}+n \alpha_{1}$ have norm 2, they are real and so have multiplicity 1 . It can also be shown from the structure of $s \hat{l}_{2}$ that the roots $n \delta=n\left(\alpha_{0}+\alpha_{1}\right)$ of norm 0 have multiplicity 1 . For the left hand side of (2), we need an explicit expression for the Weyl group $W=\left\langle r_{0}, r_{1}\right\rangle$. In fact $W \cong \mathbf{Z}_{\mathbf{2}} \propto \mathbf{Z}$, where $\left\langle r_{1}\right\rangle \cong \mathbf{Z}_{2}$ and $\left\langle r_{0} r_{1}\right\rangle \cong \mathbf{Z}$. Furthermore

$$
\left(r_{0} r_{1}\right)^{n}(\rho)=\rho+2 n \alpha_{1}-\left(n+2 n^{2}\right) \delta
$$

and

$$
r_{1}\left(r_{0} r_{1}\right)^{n}(\rho)=\rho-(2 n+1) \alpha_{1}-\left(n+2 n^{2}\right) \delta .
$$

So letting $u=e\left(-\alpha_{0}\right)$ and $v=e\left(-\alpha_{1}\right)$, for the denominator formula of $s \hat{l}_{2}$, we get:

$$
\sum_{j \in \mathbf{Z}}(-1)^{j} u^{\frac{1}{2} j(j+1)} v^{\frac{1}{2} j(j-1)}=\prod_{n \geq 1}\left(1-u^{n} v^{n}\right)\left(1-u^{n-1} v^{n}\right)\left(1-u^{n} v^{n-1}\right),
$$

which is one of the forms of the classical Jacobi Triple Product Formula. The mysterious factors not accounted for by Macdonald are in this case $\left(1-u^{n} v^{n}\right)$, which correspond to the roots $n \delta$ of norm 0 . Indeed the affine systems defined by Macdonald correspond to the real roots of affine Lie algebras. Thus Kac gave a complete interpretation for the Macdonald identities.

These generalizations of the Weyl formula lead to other beautiful formulas given by Macdonald for certain powers of the Dedekind $\eta$-function [Mac].

We work this formula out in the case of $\hat{s} l_{2}$. Let $\Delta^{0}$ be the set of positive roots of $G$ and hence here of $s l_{2}$. We divide both sides of (3) by $\prod_{\alpha \in \Delta^{0}}(1-e(-\alpha))=1-v$. Then setting $q=e(-\delta)$ and using the homomorphism $\mathbf{C}\left[\left[e\left(-\alpha_{0}\right), \ldots, e\left(-\alpha_{n}\right)\right]\right] \rightarrow$ $\mathbf{C}[[q]]$ mapping $e\left(-\alpha_{i}\right)$ to 1 if $i \geq 1$ and $e\left(-\alpha_{0}\right)$ to $q$, we eventually get

$$
\eta(q)^{3}=\sum_{n \mathbf{Z}}(4 n+1) q^{4 n^{2}+n+\frac{1}{8}}
$$

where $\eta(q)=q^{\frac{1}{24}} \prod_{n=1}^{\infty}\left(1-q^{-n}\right)$. 
Similarly, we get formulas for $\eta(q)^{n}$ for all integers $n$ corresponding to the dimension of a simple finite dimensional Lie algebra. A formula also exists for $\eta(q)^{26}$, but this case cannot be accounted for by the theory of affine Lie algebras. In fact it has not yet been interpreted. It is worth mentioning that 26 also happens to be the critical dimension in string theory. The identities for powers of the Dedekind $\eta$-function were also independently found by Dyson [Dys, though he did not notice the link with Lie algebras.

An explicit formulation for the denominator formula of a generalized Kac-Moody algebra is equivalent to complete information on its roots and simple roots. However it seems very improbable that we may find such explicit data on the root system of most infinite dimensional Kac-Moody algebras which are not affine. Therefore they do not in general yield interesting identities. In order to find satisfying formulas, you need to go to the more general level of generalized Kac-Moody algebras. This is the case for the Monster Lie algebra.

4.3. The denominator formula of the Monster Lie algebra. Recall that the Monster Lie algebra is as follows:

$$
\mathcal{M}=H \oplus\left(\oplus_{(m, n) \in \mathbf{Z}^{2}} \mathcal{M}_{(m, n)}\right),
$$

where for each $(m, n) \in \mathbf{Z}^{2}, \mathcal{M}_{(m, n)}$ is isomorphic to $V(m n)$ as a module of the Monster group $M$.

As explained at the start of $\S 4$, to prove the Moonshine Conjecture we next need to find the simple roots of $\mathcal{M}$. We see from the formulation of the denominator formula (see (1) in §4.1) that if we compute it for the Monster Lie algebra $\mathcal{M}$, the left hand side of (1) will give us the desired information. This is what we will do in this section.

Since we know the dimensions of the root spaces of $\mathcal{M}$, we can easily calculate the right hand side of (1). Set $p=e^{(-1,0)}$ and $q=e^{(0,-1)}$. Since the root space $\mathcal{M}_{(m, n)}$ is isomorphic to $V(m n)$, both these spaces have the same dimension $c(m n)$. As in $\S 1$ these are the coefficients of the Fourier expansion of the normalized modular invariant $J$. Therefore for the Lie algebra $\mathcal{M}$ the right hand side of (1) is:

$$
\prod_{m \geq 1, n \in \mathbf{Z}}\left(1-p^{m} q^{n}\right)^{c(m n)} .
$$

We now regard this product not as a formal product, but we let $p$ and $q$ be the following: $p=e^{2 \pi i \tau}$ and $q=e^{2 \pi i \mu}$, where $\tau$ and $\mu$ belong to the upper half plane $\mathcal{H}$. Then using basic techniques from number theory, in particular by taking the logarithm of the above product and then using Hecke operators, Borcherds [Bor7] shows that

$$
p(j(p)-j(q))=\prod_{m \geq 1, n \in \mathbf{Z}}\left(1-p^{m} q^{n}\right)^{c(m n)}
$$

with convergence in the domain $|p|,|q|<e^{-2 \pi}$.

Let us again think of $p$ and $q$ as the above formal exponentials. The left hand side of (4) is a sum, thus suggesting that equation (4) is the denominator formula for the Monster Lie algebra $\mathcal{M}$. If this is the case, then the left hand side gives the simple roots of $\mathcal{M}$.

Let $\mathcal{A}$ be a generalized Kac-Moody algebra with the same Cartan subalgebra $H=I I_{(1,1)} \otimes \mathbf{z} \mathbf{R}$ as $\mathcal{M}$. In particular the bilinear form on $H$ is given by the matrix 
$\left(\begin{array}{cc}0 & -1 \\ -1 & 0\end{array}\right)$. We want to define the simple roots of $\mathcal{A}$ so that the left hand side of its denominator formula

$$
e^{-\rho} \sum_{w \in W}(-1)^{l(w)} w\left(e^{\rho} \sum_{\alpha} \epsilon(\alpha) e^{\alpha}\right)
$$

will coincide with the left hand side of (4). We want to show that for all $(m, n) \in \mathbf{Z}^{2}$, the root $(m, n)$ of the Lie algebra $\mathcal{A}$ has multiplicity $c(m n)$ because we can then conclude that $\mathcal{A}$ and $\mathcal{M}$ are isomorphic and hence that the simple roots of $\mathcal{M}$ are the same as those of $A$.

In order to make a reasonable choice for the simple roots of $\mathcal{A}$, we need to write the left hand side of (4) in the form of $\left(1^{\prime}\right)$. First we need to compute the Weyl group $W$ of $\mathcal{A}$. As there is no obvious way to do so, it is reasonable to assume that $\mathcal{A}$ and $\mathcal{M}$ have the same Weyl group since we wish to show that $\mathcal{A}$ and $\mathcal{M}$ are isomorphic. Knowing the Weyl group of $\mathcal{M}$ is equivalent to knowing its real roots (see $\S 2.2$ ). Since we know what the roots of $\mathcal{M}$ are, the bilinear form on $H$ implies that the only real roots or roots of positive norm of $\mathcal{M}$ are $\pm(1,-1)$. Therefore the group $W$ has order 2 and permutes $p$ and $q$. Hence we can assume $\mathcal{A}$ has a unique positive real simple root, which we choose to be $(1,-1)$.

The bilinear form on $H$ also implies that the inner product of any two imaginary roots (distinct or not) with each other is non-zero. For the same reason as before, we can reasonably assume that $\mathcal{A}$ does not have orthogonal imaginary simple roots. Hence $-\sum_{\alpha} \epsilon(\alpha) e^{\alpha}$ reduces to the sum of all the imaginary simple roots of $\mathcal{A}$.

Since $(1,-1)$ is a simple root of $\mathcal{A}$, using the definition of the Weyl vector and equating $\left(1^{\prime}\right)$ and the left hand side of (4), we find that under the assumption that $\mathcal{A} \cong \mathcal{M}$, the elements $(1, n)$ are the simple roots of $A$ with restricted simple root multiplicity (see $\S 2.2) c(n)$. Here the multiplicity of the root $(1, n)$ equals its restricted simple root multiplicity. As mentioned earlier, this is not always the case Ray2.

Conversely since $\mathcal{A}$ is a generalized Kac-Moody algebra with Cartan subalgebra $I I_{(1,1)} \otimes_{\mathbf{Z}} \mathbf{R}$ whose simple roots are as described previously, the left hand side of the denominator formula for $\mathcal{A}$ is given by the left hand side of (4). Applying the definition of the denominator formula, we obtain that for $\mathcal{A}$ it is:

$$
p(j(p)-j(q))=\prod_{m \geq 1, n \in \mathbf{Z}}\left(1-p^{m} q^{n}\right)^{\operatorname{mult}(m, n)} .
$$

Comparing with equation (4), we conclude that the $\operatorname{root}(m, n)$ of $\mathcal{A}$ has multiplicity $c(m n)$ and so the Monster Lie algebra $\mathcal{M}$ is isomorphic to $\mathcal{A}$. The simple roots of $\mathcal{M}$ are therefore the vectors $(1, n)$ of $I I_{(1,1)}$ with multiplicity $c(n)$, and (4) gives the denominator formula for $\mathcal{M}$.

In the case of the Monster Lie algebra the denominator formula gives a product expansion of the automorphic form $j(\tau)-j(\mu)$ on $\mathcal{H} \times \mathcal{H}$. More generally, the denominator formula of various generalized Kac-Moody algebras is a product expansion of an automorphic form.

4.4. Product expansions of automorphic forms on Grassmannians. In [Bor10], Borcherds gives sufficient conditions for an automorphic form on a Grassmannian to have a product expansion. In some cases, these expansions correspond to the denominator formula of a generalized Kac-Moody algebra or more generally 
of a generalized Kac-Moody superalgebra (see $\S 6$ below). We shall discuss this in some detail in this section.

Before defining automorphic forms on Grassmannians, we remind the reader of the definition of a Grassmannian. Let $M$ be a non-singular even lattice. We restrict ourselves to the case when the lattice $M$ has an integral bilinear form (.,.), and the real space $M \otimes \mathbf{z} \mathbf{R}$ has dimension $s+2$ and signature $2-s$. Maximal positive definite subspaces of $M \otimes_{\mathbf{z}} \mathbf{R}$ have dimension 2. Choose a maximal positive definite subspace and a basis $e_{1}, e_{2}$ of it. Any basis $v_{1}, v_{2}$ of a maximal positive definite subspace is said to be positively oriented if $\left(e_{1} \wedge e_{2}, v_{1} \wedge v_{2}\right)>0$. Thus there are two distinct orientations on $M$.

The Grassmannian $G(M)$ is the set of oriented positive definite 2-dimensional subspaces of $M \otimes_{\mathbf{z}} \mathbf{R}$. In other words, $G(M)$ is the set of all exterior products $v_{1} \wedge v_{2}$, where $v_{1}, v_{2}$ is a positively oriented orthonormal basis of a positive definite subspace of $M$.

There is another useful construction of $G(M)$. Let $P$ be the set of norm 0 vectors $x+i y \in M \otimes \mathbf{C}$ such that $(x, y)$ is a positively oriented orthogonal basis. Choose a norm 0 primitive vector $z$ in $M$ (i.e. for any integer $n>1, \frac{z}{n} \notin M$ ). Let $z^{*}$ be in the dual $M^{*}$ satisfying $\left(z, z^{*}\right)=1$. Consider the lattice $L=\left(M \cap z^{\perp}\right) / \mathbf{Z} z$. The lattice $L$ is Lorentzian (see $\S 2.4$ ). So the positive norm vectors of $L \otimes \mathbf{R}$ form two open cones. We let $\mathcal{C}$ be the cone of positive norm vectors $y \in L \otimes \mathbf{R}$ satisfying $\left(y, y_{M}\right)>0$, where $\left(x_{M}, y_{M}\right)$ is a positively oriented basis such that $\left(y_{M}, z\right)=0$ and $\left(x_{M}, z\right)>0$. For $v \in L \otimes \mathbf{C}, a, b \in \mathbf{C}$, let $(v, a, b)$ denote the vector $v+a z^{*}+b z$ of $M \otimes \mathbf{C}$. Since the vector space $L \otimes \mathbf{R}$ is isomorphic to the subspace of $M \otimes \mathbf{R}$ of vectors orthogonal to both $z$ and $z^{*}$, it follows that $L \otimes \mathbf{R}+i \mathcal{C}$ can be identified with the subset $\left\{\left(v, 1,-v^{2} / 2-z^{*} / 2\right) \mid v \in L \otimes \mathbf{C}\right\}$ of vectors in $P$ having inner product 1 with $z$. This set is in bijective correspondence with the Grassmannian $G(M)$. Therefore $G(M)$ can be taken to be $L \otimes \mathbf{R}+i \mathcal{C}$.

All elements of $O_{M}(\mathbf{R})$ are products of reflections with respect to vectors of $M \otimes \mathbf{R}$ of non-zero norm, and $O_{M}(\mathbf{R})^{+}$is the subgroup of elements whose decomposition into reflections contains an even number of reflections with respect to vectors of negative norm. The group $O_{M}(\mathbf{R})^{+}$acts on $P$ and on $G(M)$.

An automorphic form of weight $k$ on $G(M)$ for a subgroup $A$ of finite index of $O_{M}(\mathbf{Z})^{+}$and a one dimensional representation $\chi$ of $A$ is a real valued function $\Phi$ on $P$ satisfying

$$
\Phi(v z)=z^{-k} \Phi(v), v \in P, z \in \mathbf{C}, \quad \text { and } \quad \Phi(g z)=\chi(g) \phi(z) .
$$

It can be shown that an automorphic form $\Phi$ is fully determined by its restriction on the image of $L \otimes \mathbf{R}+i C$ in $P$.

We now explain Borcherds' result on product expansions of automorphic forms in its most general form. To do this, we need to first define a modular form with values in a vector space, which generalizes the usual notion of a complex valued modular form.

We remind the reader that the metaplectic group

$$
M p_{2}(\mathbf{Z})=\left\{\left(\left(\begin{array}{ll}
a & b \\
c & d
\end{array}\right), \pm \sqrt{c \tau+d}\right) \mid\left(\begin{array}{ll}
a & b \\
c & d
\end{array}\right) \in S L(2, \mathbf{Z}), \tau \in \mathcal{H}\right\}
$$

is a double cover of $S L(2, \mathbf{Z})$. The group multiplication is given by

$$
(A, f(\tau))(B, g(\tau))=(A B, f(B(\tau)) g(\tau)),
$$


where $f$ and $g$ are functions on $\mathcal{H}$ of the form $\pm \sqrt{c \tau+d}$. The group $M p_{2}(\mathbf{Z})$ is generated by the elements $S=\left(\left(\begin{array}{cc}0 & -1 \\ 1 & 0\end{array}\right), \sqrt{\tau}\right)$ and $T=\left(\left(\begin{array}{cc}1 & -1 \\ 0 & 1\end{array}\right), 1\right)$. Let $\rho$ be a representation of $M p_{2}(\mathbf{Z})$ on the complex vector space $V$. Then a real analytic function $F: \mathcal{H} \mapsto V$ is an almost holomorphic modular form of weight $\left(k^{+}, k^{-}\right)$for $k^{ \pm} \in \frac{1}{2} \mathbf{Z}$ and type $\rho$ on $\mathcal{H}$ if

$$
F\left(\frac{a \tau+b}{c \tau+d}\right)=(c \tau+d)^{k^{+}}(c \bar{\tau}+d)^{k^{-}} \rho\left(\left(\begin{array}{ll}
a & b \\
c & d
\end{array}\right), \pm \sqrt{c \tau+d}\right) F(\tau)
$$

for any element $\left(\left(\begin{array}{ll}a & b \\ c & d\end{array}\right), \pm \sqrt{c \tau+d}\right) \in M p_{2}(\mathbf{Z})$ and the complex valued functions $f_{\alpha}$ on $\mathcal{H}$ defined by $F=\sum f_{\alpha} v_{\alpha}$, where the vectors $\left.\left\{v_{\alpha}\right\}\right|_{\alpha}$ form a basis of $V$, can be written as

$$
\sum_{r \in \mathbf{Q}} \sum_{s \in \mathbf{Z}} c(r, s) e^{2 \pi r i \tau}(\operatorname{Im}(\tau))^{-s}
$$

Therefore $F$ is holomorphic on $\mathcal{H}$ if $k^{-}=0$ and the coefficients $c(r, s)=0$ for $s \neq 0$.

Note that we recover the usual definition of a complex valued holomorphic modular function for $S L(2, \mathbf{Z})$ with character $\chi$ when $V=\mathbf{C}, k^{-}=0$ and $\rho$ is the representation of $M p_{2}(\mathbf{Z})$ induced from the representation of $S L(2, \mathbf{Z})$ given by $\chi$. More generally a holomorphic modular form of level $N$ for a subgroup of $S L(2, \mathbf{Z})$ induces a modular form of the above type. Jacobi forms EicZ are also examples of vector valued modular forms.

The automorphic forms which can be shown to have product expansions are constructed from modular forms with values in a certain vector space and of a certain type. In this paragraph we give more details about these modular forms. Let $M^{*}$ be the dual of $M$. Then $M \subset M^{*}$ and $M^{*} / M$ is a finite abelian group of order $\operatorname{det} M$. We consider the group ring $V=\mathbf{C}\left[M^{*} / M\right]$ as a vector space with basis $e_{\alpha}$ for $\alpha \in M^{*} / M$ and the representation $\rho$ of $M p_{2}$ on $V$ given by

$$
\rho(S)\left(e_{\alpha}\right)=\frac{\sqrt{i}^{k^{-}-k^{+}}}{\sqrt{\left|M^{*} / M\right|}} \sum_{\gamma \in M^{*} / M} e^{-2 \pi i(\alpha, \gamma)} e_{\gamma}
$$

and

$$
\rho(T)\left(e_{\alpha}\right)=e^{\pi i(\alpha, \alpha)} e_{\alpha}
$$

We are now ready to give a rough idea of Borcherds' main result on product expansions of automorphic forms. From a modular form $F$ of weight $(1-s / 2,0)$ and type $\rho$, holomorphic on $\mathcal{H}$ whose components $f_{\alpha}, \alpha \in M^{*} / M$ have integer coefficients $c_{\alpha}(r)$ for all $r \leq 0$, Borcherds constructs an automorphic form $\Phi$ on the Grassmannian $G(M)$ and gives its Fourier expansion. He then shows that whenever the norm 0 vector $z$ is primitive, its restriction $\Phi_{z}$ to $L \otimes \mathbf{R}+i C$, where $L=M \cap z^{\perp} / \mathbf{Z} z$ (see above), has a converging product expansion in a region of $L \otimes \mathbf{R}+i C$.

We explain this in greater detail in the special case when $M$ is even unimodular Bor8, i.e. $M=M^{*}$. Such a lattice can be written

$$
M=K \oplus I I_{1,1} \oplus I I_{1,1},
$$

where $I I_{1,1}$ is the even unimodular Lorentzian lattice of rank 2 (see $\S 3.5$ ) and $K$ is a positive definite lattice in $M$. We remind the reader that the theta function of $K$ 
is the complex valued modular function on $\mathcal{H}$ with the following Fourier expansion:

$$
\theta_{K}(\tau)=\sum_{\alpha \in K} q^{(\alpha, a) / 2}
$$

where as usual $q=e^{2 \pi i \tau}$. The lattice $L=K \oplus I I_{1,1}$ is an even unimodular Lorentzian lattice which is isomorphic to $M \cap z^{\perp} / \mathbf{Z} z$ for any primitive vector $z$ of norm 0 . Let $f(\tau)=\sum_{n \in \mathbf{Z}} a(n) q^{n}$ be a complex valued modular form, holomorphic on $\mathcal{H}$ of weight $-s / 2$ with integer coefficients $a(n)$, and $24 \mid a(0)$ when $s=0$. Let $d$ be the constant term of $\theta_{K}(\tau) f(\tau)$ and $m$ the constant term of $\theta_{K}(\tau) f(\tau) E_{2}(\tau)$, where $E_{2}(\tau)$ is the Eisenstein series of weight 2: $E_{2}(\tau)=1-2 \sum_{n>0}\left(\sum_{l \mid n} l\right) q^{n}$. Then

$$
\Phi(y)=e^{-2 \pi i(\rho, y)} \prod_{r \in M,(r, y)>0}\left(1-e^{-2 \pi i(r, y)}\right)^{a(-(r, r) / 2)},
$$

where $y \in L \otimes \mathbf{R}+i \mathcal{C}$ and $\rho=\left(\sum_{(r, y)>0} a(-(r, r) / 2) r / 2, m, d / 24\right)$ is an element of $L$, is a meromorphic automorphic form of weight $a(0) / 2$ for $O_{M}(\mathbf{Z})^{+}$. Furthermore if $y \in K \otimes \mathbf{C}$ is a zero or a pole of $\Phi$, then there exists an element $x$ in $M$ of positive norm orthogonal to $y$. The element $x$ can be written $(b, 2 a, c)$ with respect to the standard basis of $I I_{1,1}$, where $b \in L, a, c \in \mathbf{Z}$ such that $(b, b)-4 a c>0$. With this notation, if $y$ is a zero, then the multiplicity of $y$ is $\sum_{n>0} a\left(n^{2}(2 a c-(b, b))\right)$.

The simplest application of this result gives an alternative proof for the denominator formula of the Monster Lie algebra. With $K=0$ and $f$ equal to the normalized modular invariant $J$, the above implies that the right hand side of (4) in $\S 4.3$ is an automorphic form. Then the formula for the multiplicities of zeros leads to equality (4).

We also get the denominator formula of the fake Monster Lie algebra from the above result. Nikulin and Gritsenko use this work of Borcherds in their study of hyperbolic Lie algebras contained in generalized Kac-Moody algebras or superalgebras in such a way that both have the same real root spaces. In particular, they give explicit product expansions of automorphic forms connected to denominator formulas in these cases GriN1, GriN2, GriN3].

There are other product expansions of automorphic forms which correspond to denominator formulas of generalized Kac-Moody algebras or superalgebras. However this is not a general rule. For example, if the lattice $M$ is Lorentzian of rank at least 3, then Nikulin and Gritsenko have shown that for each rank there are only finitely many product expansions corresponding to generalized Kac-Moody algebras or superalgebras [Nik2].

In Bor9] Borcherds also gives sufficient conditions for a meromorphic modular function to have a product expansion. Let $\Psi(\tau)$ be a meromorphic modular form for some character of $S L(2, \mathbf{Z})$, of weight $k$, leading coefficient 1 , whose zeros and poles are imaginary quadratic irrationals or cusps. Then there exists a modular form

$$
f(\tau)=\sum_{n \in \mathbf{Z}} a(n) q^{n}
$$

holomorphic on $\mathcal{H}$ of weight $1 / 2$ and level 4 with integral coefficients which are non-zero only if $n \equiv 0 \quad$ or $1(\bmod 4)$ such that

$$
\Psi(\tau)=q^{h} \prod_{n>0}\left(1-q^{n}\right)^{a\left(n^{2}\right)}
$$


and $k=a(0)$. The constant $h$ is the constant term of $-f(\tau) \sum H(n) q^{n}$, where $H(n)$ is the Hurwitz class number of $n$. Conversely for any such modular function $f$, there exists a modular form $\Psi$ with the above product expansion.

For example, the classical $\Delta$ function satisfies the above conditions for $\Psi(\tau)$, and its product expansion $\Delta(\tau)=q \prod_{n>0}\left(1-q^{n}\right)^{24}$ corresponds to $f(\tau)=$ $12+24 \sum_{n>0} q^{n^{2}}$. The modular invariant $j$ also satisfies these conditions and thus its product expansion corresponds to some $f$. This is also the case for the product expansions of the Eisenstein series $E_{4}, E_{6}, E_{8}, E_{10}, E_{12}$.

The product expansion of some of the Eisenstein series, for example $E_{6}$, corresponds to the denominator formula of a generalized Kac-Moody algebra. Again various product expansions of modular forms have an interpretation in terms of generalized Kac-Moody algebras or superalgebras (see below), but this does not seem to be the case for most of them (as, for example, $\Delta, j, E_{4}$ ).

We saw in $\S 4.2$ that the denominator formulas of the affine Lie algebras give the Macdonald identities. They are product expansions of Jacobi forms for $S L(2, \mathbf{Z})$. Jacobi forms play a fundamental role in the theory of affine Lie algebras [KacP3]. In Bor9, Borcherds gives sufficient conditions for other products to be Jacobi forms. We finish this section by giving a brief summary of this result.

A Jacobi form of weight $k$ and index $m$ EicZ is a holomorphic function $\phi$ : $\mathcal{H} \times \mathbf{C} \rightarrow \mathbf{C}$ such that

$$
\phi\left(\frac{a \tau+b}{c \tau+d}, \frac{z}{c \tau+d}\right)=(c \tau+d)^{k} e^{\frac{2 \pi i m c z^{2}}{c z+d}} \phi(\tau, z)
$$

for $\left(\begin{array}{ll}a & b \\ c & d\end{array}\right) \in S L(2, \mathbf{Z})$. Then $\phi$ can be considered as a function of $q=e^{2 \pi i \tau}$ and $\zeta=e^{2 \pi i z}$ and has a Laurent expansion $\sum a(n, m) q^{n} \zeta^{m}$.

Let $K$ be a positive definite integral lattice. We give a generalization of the notion of an indecomposable finite root system. Let $c$ be function $E \rightarrow \mathbf{Z}_{+}$such that $c(v) \neq 0$ for only finitely many vectors $v \in K$, satisfying $c(v)=c(-v)$ and for which there exists $r \in \mathbf{R}$ such that for any element $y \in K \otimes \mathbf{R}$ of norm, $\sum_{v \in K} c(v)(y, v)^{2}=r$. The multiset $V$ containing $c(v)$ copies of each vector $v$ of $K$ is called a vector system. We shall use the same notation $v$ for each copy of $v$. Set $d$ to be the number of elements in $V$ (counted with multiplicity). When $c(E) \subset\{0,1\}$ and $V$ is indecomposable, $c$ is a finite root system.

As in the case of root systems, the hyperplanes perpendicular to $V$ partition $E$ into Weyl chambers. Let $W$ be a fixed Weyl chamber. Let $w$ be a vector in the interior of $W$. A vector $v \in V$ is said to be positive (resp. negative) if $(v, w)>0$ (resp. <0). All vectors in $V$ are either positive or negative irrespective of the choice of $w$. The Weyl vector is defined as follows: $\rho=\frac{1}{2} \sum_{v \in V, v>0} v$. For $v \in V$, $n \in \mathbf{Z}$, we write $(v, n)>0$ if either $n>0$ or $n=0$ and $v>0$. Borcherds showed that

$$
\phi(\tau, z)=q^{\frac{d}{24}} \zeta^{-\rho} \prod_{v \in V, n \in \mathbf{Z},(v, n)>0}\left(1-q^{n} \zeta^{v}\right)
$$

is a sum of theta functions times modular forms holomorphic on $\mathcal{H}$ and thus is a Jacobi form. The Macdonald identities, i.e. the denominator formula for the untwisted affine Lie algebras, correspond to the case when $V$ is an indecomposable root system. 
4.5. The character formula and Lie algebra homology. Coming back to the proof of the Moonshine Conjecture, we now know the exact structure of the Monster Lie algebra. As explained in $\S 1.3$, we next need to find formulas which will give all the coefficients of the Thompson series $T_{g}, g \in M$. This will finally allow us to conclude that these series are the same as the Hauptmoduls given by Norton and Conway. In order to find these formulas, Borcherds uses the homology theory of generalized Kac-Moody algebras. This section will be devoted to explaining this theory and applying it to the Monster Lie algebra.

Let $G$ be a generalized Kac-Moody algebra and $F$ the subalgebra generated by the elements $f_{i}, i \in I$. We keep the notation of previous chapters. Let $V(\Lambda)^{t}$ be the right $G$-module with the same underlying vector space as $V(\Lambda)$ and with the canonical $G$-action given by the $G$-action on $V(\Lambda)$.

The character formula for $V(\Lambda)$ can be deduced from the Euler-Poincaré principle applied to the homology of $F$ with values in $V(\Lambda)^{t}$ and a straightforward generalization to generalized Kac-Moody algebras of the Garland-Lepowsky proof of Kostant's formula in the Kac-Moody algebra case GarL. More precisely, let $S$ be a subset of $I$ and $G_{S}$ the generalized Kac-Moody algebra associated to the ma$\operatorname{trix} A_{S}=\left(a_{i j}\right)_{i, j \in S}$ and with Cartan subalgebra $H$. Let $\Delta_{+}^{S}$ be the set of positive roots of $G_{S}$ and $N_{S}^{-}$the Lie subalgebra generated by the negative root spaces of $G_{S}: N_{S}^{-}=\oplus_{\alpha \in \Delta_{s}^{+}} G_{-\alpha}$. The standard $G_{S^{-}}$module Koszul complex:

$$
\ldots \longrightarrow V(\Lambda)^{t} \otimes \Lambda^{j}\left(N_{S}^{-}\right) \longrightarrow V(\Lambda)^{t} \otimes \Lambda^{j-1}\left(N_{S}^{-}\right) \longrightarrow \ldots \longrightarrow V(\Lambda) \longrightarrow 0,
$$

with the usual maps gives the homology groups $H_{j}\left(N_{S}^{-}, V(\Lambda)\right)$.

Since the root spaces of $N_{S}^{-}$and the weight spaces of $V(\Lambda)$ are finite dimensional and the weights of $V(\Lambda)^{t} \otimes \Lambda^{j}\left(N_{S}^{-}\right)$are of type $\Lambda-\sum k_{i} \alpha_{i}, k_{i} \in \mathbf{Z}_{+}$, the Euler-Poincaré principle holds: Let $\Lambda\left(N_{S}^{-}\right)$and $H\left(N_{S}^{-}, V(\Lambda)\right)$ be the virtual spaces $\sum(-1)^{i} \Lambda^{i}\left(N_{S}^{-}\right)$and $\sum(-1)^{i} H_{i}\left(N_{S}^{-}, V(\Lambda)\right)$ respectively. Then

$$
\Lambda\left(N_{S}^{-}\right) \otimes V(\Lambda)=H\left(N_{S}^{-}, V(\Lambda)\right) .
$$

It can be shown that as a $G_{S^{-}}$-module, $H_{i}\left(N_{S}^{-}, V(\Lambda)\right)$ is the sum of all the $G_{S^{-}}$ submodules of $\Lambda^{i}\left(N_{S}^{-}\right) \otimes V(\Lambda)$ isomorphic to the irreducible $G_{S}$-module $S(\lambda)$ for $\lambda \in H^{*}$ satisfying the following: $\left(\lambda, \alpha_{i}\right) \geq 0$ for all $i \in S$ and $\frac{2\left(\lambda, \alpha_{i}\right)}{\left(\alpha_{i}, \alpha_{i}\right)} \in \mathbf{Z}$ if $i \in S$ and $a_{i i}>0$ and $(\lambda+\rho, \lambda+\rho)=(\Lambda+\rho, \Lambda+\rho)$ and $\Lambda-\lambda$ is the sum of $i$ roots in $\Delta_{+}-\Delta_{+}^{S}$. A root $\lambda \in H^{*}$ has this property if and only if

$$
\lambda=w\left(\rho+\Lambda-\sum_{j=1}^{s} \alpha_{i_{j}}\right)-\rho
$$

where the simple roots $\alpha_{i_{j}}$ are mutually orthogonal and each is orthogonal to $\Lambda$ and the element $w \in W$ has length $i-r$.

When $G$ is a Kac-Moody algebra and $G_{S}$ is a finite dimensional Lie algebra, the above was proved by Garland and Lepowsky GarL] and for arbitrary subsets $S$ of $I$ by Liu [Liu]. This result was extended to generalized Kac-Moody algebras by Naito [Nai].

We apply the above to the particular case when $S=\emptyset$ :

$$
H(F, V(\Lambda))=\oplus V\left(w\left(\rho+\Lambda-\sum \alpha_{i_{j}}\right)-\rho\right)
$$

shows that equality (5) is equivalent to the character formula for the highest weight module $V(\Lambda)$. Consider the virtual space $\Lambda(F)$. This is defined as before as the 
alternate sum $\sum(-1)^{i} \Lambda^{i}(F)$. Its character is easily seen to be

$$
\prod_{\alpha \in \Delta_{+}}\left(1-e^{-a}\right)^{\text {mult } \alpha}
$$

by considering $F$ as a sum of 1-dimensional spaces since $\Lambda(U \oplus V)=\Lambda(U) \otimes \Lambda(V)$ for any vector spaces $U, V$.

When $S=\emptyset$ and $\Lambda=0$, we get the homology $H(F)$ of $F$, and

$$
\Lambda(F)=H(F)
$$

is equivalent to the denominator formula for $G$. Equality (6) in this case is known as Kostant's formula, which he proved for finite dimensional simple Lie algebras. Using these formulas when $\Lambda=0$, Kang [Kan1], Kan2] proved a recursive formula and a closed form formula for the root multiplicities of Kac-Moody algebras, from which he deduces a formula for the weight multiplicities of irreducible integrable highest weight modules. Applying his results to the Monster Lie algebra, he discovered many interesting identities between the coefficients of the modular invariant $j$ Kan2 and for arbitrary Thompson series KacK. This was also done by Jurisch, Lebowsky and Wilson JurLW. Kang's work should also lead to the structure of $G$ considered as a $G_{S}$-module. Note that this $G_{S}$-module not only is not a highest or lowest weight module but does not altogether belong to the category $\mathcal{O}$ of integrable $G_{S}$-modules $V$ defined to be $V=\sum V(\lambda)$, where $V(\lambda)$ is a finite dimensional $H$-weight space and there is a finite number of weights $\nu_{1}, \ldots, \nu_{s}$ such that if $\lambda$ is a weight of $V$, then there exists $i$ for which $\nu_{i}-\lambda$ is a positive sum of simple roots of $G_{S}$. Very little is known about generalized Kac-Moody algebra representations not belonging to this category.

It is straightforward to apply the above results to the Monster Lie algebra since the Weyl group in this case has order 2. We find:

$$
p^{-1} \Lambda\left(\sum_{m>0, n \in \mathbf{Z}} V_{m n} p^{m} q^{n}\right)=\sum_{m} V(m) p^{m}-\sum_{n} V(n) q^{n},
$$

where $\Lambda\left(\sum_{m>0, n \in \mathbf{Z}} V_{m n} p^{m} q^{n}\right)$ again denotes an alternate sum of exterior powers. Since the vector spaces $V(n)$ are finite dimensional, the Adams operations $\phi^{i}$ and the splitting principle in the trivial case of vector bundles over $\mathbf{C}$ (ㅅi] $)$ give:

$$
\begin{array}{r}
p^{-1} \exp \left(-\sum_{i>0} \sum_{m>0, n \in \mathbf{Z}} \operatorname{Tr}\left(g^{i} \mid V(m n)\right) p^{m i} q^{n i} / i\right) \\
=\sum_{m} \operatorname{Tr}(g \mid V(m)) p^{m}-\sum_{n} \operatorname{Tr}(g \mid V(n)) q^{n}
\end{array}
$$

for any element $g$ in the Monster group $M$.

4.6. Replicable functions. Equation (7) of $\S 4.5$ is the formula for the Thompson series we were aiming for. It induces various formulas for the coefficients of these series, and in particular it implies that all the coefficients can be determined from the first few ones. In this final section related to the proof of the Moonshine Conjecture, we give some details about this last step.

Any formal power series satisfying an equation such as (7) is said to be completely replicable. We next explain in greater detail what this means. Equation (7) says that for all $g \in M$, the Thompson series $T_{g}(q)=\sum_{n} \operatorname{Tr}(g \mid V(n)) q^{n}$ is a replicable function. 
In [ConN], by considering the Hecke transform $T(p) J$ of the normalized modular invariant $J$, Conway and Norton showed that for any prime $p, J^{p}(q)-J\left(q^{p}\right)$ equaled a polynomial in positive powers of $q$ with integral coefficients. They found that similar equations seem to hold for $T_{g}^{p}(q)-T_{g^{p}}\left(g^{p}\right)$ for any element $g \in M$ and that $p$ could also be taken to be a composite integer. These formulae, which they called the replication formulae, yield many identities for the coefficients of the series $T_{g}$. Replicable functions were defined formally by Norton [Nor1] in 1984. We reproduce the definition given by Alexander, Cummins, McKay and Simons in AleCMcKS, which is equivalent to Norton's original one.

Let

$$
f(q)=q^{-1}+\sum_{m=1}^{\infty} a_{m} q^{m}
$$

be a formal power series with integer coefficients. For each positive integer $n$, there exists a unique monic polynomial $P_{n}(t)$ with integral coefficients for which $P_{n}(f(q))-q^{-n}$ is a series in positive powers of $q$. The series $f(q)$ is a replicable function if for all positive integers $n$, there is a power series

$$
f^{(n)}=q^{-1}+\sum_{m=1}^{\infty} a_{m}^{(n)} q^{m}
$$

called the $n$-th replicate of $f(q)$ such that

$$
\frac{1}{n} \sum_{a d=n, 0 \leq b<d} f^{(a)} \exp \left(\left(2 \pi i \frac{b}{d}\right) q^{\frac{a}{d}}\right)=\frac{1}{n} P_{n}(f(q)) .
$$

When for all $a, f^{(a)}(q)=f(q)$, the left hand side of (8) reduces to the Hecke transform $T(n) f(q)$ of $f(q)$. This is the case for $J(q)$. It follows from $(7)$ that the $n$-th replicate of the Thompson series $T_{g}(q)$ is $T_{g^{n}}(q)(g \in M)$.

Norton had conjectures in Nor1 that a function $f(q)$ of the above type with infinitely many non-zero coefficients is replicable if and only if $f(q)$ is a normalized Hauptmodul for a genus 0 subgroup $G$ of $S L(2, \mathbf{R})$ containing the congruence subgroup

$$
\Gamma_{0}(N)=\left\{\left(\begin{array}{ll}
a & b \\
c & d
\end{array}\right) \in S L(2, \mathbf{R} \mid c \equiv 0(\bmod N)\}\right.
$$

for some positive integer $N$. In [CumN], Cummins and Norton proved that a normalized Hauptmodul for a genus 0 subgroup $\Gamma_{0}(N) \leq G \leq S L(2, \mathbf{R})$ is replicable. To prove the converse, more conditions are necessary.

Since the replicates of $T_{g}(q)$ are $T_{g^{n}}(q)$, all the replicates of $T_{g}(q)$ are replicable and for all $n, m \in \mathbf{N}$,

$$
T_{g}^{(n)(m)}(q)=T_{g}^{(n m)}(q) .
$$

Any replicable function with such properties is said to be completely replicable. This property is equivalent to equality $(7)$ in $\S 4.5$.

It can be shown that all the coefficients of a completely replicable function are determined by the first few coefficients. To complete the proof of the Moonshine Conjecture, Borcherds uses a result by Koike [Koi] which shows that the Hauptmoduls given by Conway and Norton are completely replicable. Therefore it is only a matter of checking whether these Hauptmoduls and the Thompson series given by 
Frenkel, Lepowsky and Meurman's action of the Monster on the moonshine module coincide by considering their first few coefficients. We give two examples:

1. For $g=1$, we already saw that $T_{g}=J$ and the corresponding group of genus 0 is $S L(2, \mathbf{Z})$ itself.

2. When $g$ is an involution belonging to the conjugacy class $2 B$ in the notation of the Atlas of character tables of finite simple groups ConN, $T_{g}(q)=$ $24+q^{-1} \prod_{n>0}\left(1-q^{2 n+1}\right)^{24}$ and the genus 0 group is the principal congruence subgroup $\Gamma_{0}(2)$.

As mentioned in $\S 1.3$, rather than doing a case by case study to check that the list of Hauptmoduls given by Conway and Norton coincides with the Thompson series, one can use a result of Cummins and Gannon CumG. Since $M$ is a finite group, for any $g \in M, T_{g}(q)$ has only finitely many distinct replicates, and it can be easily verified that $T_{g}(q)$ has infinitely many non-zero coefficients. It can be shown that a completely replicable function with such properties satisfies a modular equation of degree $p$ for infinitely many primes $p$. By this we mean that there are polynomials $F_{p}(x, y) \in \mathbf{C}[x, y]$ of degree $p$ in $x$ and $y$, which are monic in $x$ and $y$ and such that

$$
F_{p}(x, y)=F_{p}(y, x) \text { and for all } 0 \leq b<p, F_{p}\left(f(q), f\left(\exp \left(2 \pi i \frac{b}{p}\right) q^{\frac{1}{p}}\right)\right)=0,
$$

as a formal $q$-series. Cummins and Gannon's result implies that functions with this property are Hauptmodul for genus 0 subgroups of $S L(2, \mathbf{R})$.

We have finished explaining the proof of the Moonshine Conjecture. The following two sections discuss two interesting topics related to the field of generalized Kac-Moody algebras.

\section{LiE SUPERALGEBRAS}

5.1. Definitions and elementary properties. A Lie superalgebra is a $\mathbf{Z}_{2}$-graded algebra $G=G_{\overline{0}} \oplus G_{\overline{1}}$ with a Lie bracket satisfying

$$
[a, b]=-(-1)^{|a b|}[b, a] \quad \text { and } \quad[a[b, c]]=[[a, b] c]+(-1)^{|a b|}[[a, c] b],
$$

where for any homogeneous element $x \in G_{\bar{n}}, n=0,1,|x|=\bar{n}$. The subspace $G_{\overline{0}}$ (resp. $G_{\bar{T}}$ ) is the even (resp. odd) part of $G$. The finite dimensional simple Lie superalgebras were classified by Kac [Kac3] in the 70s. Kac also defined the KacMoody superalgebras and proved a character formula for their highest weight modules [Kac4]. The class of generalized Kac-Moody algebras can also be extended to that of generalized Kac-Moody superalgebras [Ray1], Ray3, and natural examples of generalized Kac-Moody superalgebras which are not Kac-Moody superalgebras occur in the context of the Moonshine Conjecture [Bor7].

Let $I$ be the indexing set as in $\S 2$, and $I_{1}$ a subset indexing the odd generators. Apart from the defining properties given in the case of a generalized Kac-Moody algebra, the Cartan matrix also satisfies $\frac{a_{i j}}{a_{i i}} \in \mathbf{Z}$ if $a_{i i}>0$ and $i \in I_{1}$. The Cartan subalgebra is assumed to be in $G_{\overline{0}}$.

Apart from the relations given in $\S 2$, the generators $e_{i}, f_{i}, i \in I$, and $H$ satisfy also:

$$
\begin{gathered}
\operatorname{deg} e_{i}=\operatorname{deg} f_{i}= \begin{cases}\overline{0}, & \text { if } i \notin I_{1} \\
\overline{1}, & \text { if } i \in I_{1}\end{cases} \\
\left(\operatorname{ad} e_{i}\right)^{\frac{-a_{i j}}{a_{i i}}+1} e_{j}=0=\left(\operatorname{ad} f_{i}\right)^{\frac{-a_{i j}}{a_{i i}}+1} f_{j}, \quad \text { for } \quad a_{i i}>0 \text { and } i \in I_{1} .
\end{gathered}
$$


We give some surprising properties which make Lie superalgebras more complex structures than simple generalizations of Lie algebras.

Unlike the Lie algebra case, most of the simple finite dimensional Lie superalgebras are not generalized Kac-Moody superalgebras. In fact not all of them are contragredient Lie superalgebras (i.e. associated to a "Cartan" matrix). Only the classical ones are, by which we mean the finite dimensional simple Lie algebras and the Lie superalgebras of type $A(m, n), B(m, n), C(n), D(m, n), D(2,1 ; \alpha)$ for $\alpha \neq 0,-1, F(4)$, and $G(3)$ (see $[\mathrm{Kac} 3$ ). We use the standard symbols introduced by Kac in Kac3. A simple classical Lie superalgebra $G$ is a generalized KacMoody superalgebra if and only if $G$ is of type $A(m, 0), A(m, 1), B(0, n), B(m, 1)$, $C(n), D(m, 1), D(2,1 ; \alpha)$ for $\alpha \neq 0,-1, F(4)$, and $G(3)$. Affine Lie superalgebras are constructed from the classical Lie superalgebras in a manner similar to affine Lie algebras. Very few of them are generalized Kac-Moody superalgebras: apart from the affine Lie algebras, only those of type $A^{(2)}(0,2 n-1), n \geq 3, A^{(2)}(0,3)$, $C^{(2)}(n+1), n \geq 2, C^{(2)}(2), A^{(4)}(0,2 n), n \geq 2$, and $A^{(4)}(0,2)$ Kac4, Leu, Ray3. are.

Also Borcherds' above characterization in terms of an almost positive definite bilinear form has little meaning for superalgebras, as $A(1, n), B(0, n)$ and $B^{(1)}(0, n)$ Kac3, Kac4 are the only Lie superalgebras which are not Lie algebras satisfying this characterization [Ray3.

As we saw in $\S 2.2$, there are three equivalent definitions of real roots in the case of generalized Kac-Moody algebras. In the general context of generalized Kac-Moody superalgebras, these three definitions are no longer equivalent. For example, in the finite-dimensional Lie superalgebra $A(m, 1)$, there are roots of negative norm and roots of positive norm satisfying condition (iii) (see $\S 2.2$ ), i.e. giving rise only to finite chains through any other root. Furthermore the negative norm roots of $A(m, 1)$ are not conjugate to simple roots under the action of the Weyl group. Therefore, real and imaginary roots should be defined in terms of the length of the root chains they give rise to. For real roots, this amounts to defining them by condition (iii) of $\S 2.2$.

5.2. Character formulas of generalized Kac-Moody superalgebras. Generalized Kac-Moody superalgebras have highest weight modules, and a character formula for integrable irreducible highest weight modules of Kac-Moody superalgebras was proved by Kac [Kac4] and later generalized to generalized Kac-Moody superalgebras [Ray1, GriN1. However as most contragradient affine and finite dimensional simple Lie superalgebras are not generalized Kac-Moody superalgebras, there is no general method to find a character formula in all the integrable cases [Fru, Jeu], JeuHKT], Kac5], Serg. The problem is that the Cartan matrix can contain some non-diagonal entries which are negative and some which are positive. So one cannot apply the method used in the Lie algebra case. From the character formula of a generalized Kac-Moody superalgebra, one can deduce its denominator formula; and when it can be explicitly computed, it also corresponds to a product expansion of an automorphic form. Denominator formulas for all classical simple Lie superalgebras and their associated affine Lie superalgebras were established by Kac and Wakimoto in $\mathrm{KacW}$. As in the Lie algebra case, they give new proofs for various classical identities. 


\section{LATTICES}

6.1. Some basic facts. Since positive definite and Lorentzian lattices play a preponderant role in the theory of generalized Kac-Moody algebras, we shall finish by mentioning them in slightly greater detail.

Elements of norm 2 are called roots, and positive definite lattices generated by roots are the root lattices of the simply-laced finite dimensional Lie algebras, i.e. those of type $\mathbf{A}, \mathbf{D}, \mathbf{E}$ whose roots all have the same length. The determinant $\operatorname{det} L$ of $L$ is an invariant of $L$ given by $\left.\operatorname{det}\left(e_{i}, e_{j}\right)\right|_{i, j}$, where the elements $e_{i}$ form a basis of $L$. Let $L^{*}$ be the dual of $L$ in $L \otimes \mathbf{R}$. Then $L^{*}$ is a lattice containing $L$ and $L^{*} / L$ is an abelian group of order $\operatorname{det} L$. Hence a lattice $L$ is unimodular (i.e. $L=L^{*}$ ) if and only if $\operatorname{det} L= \pm 1$. They form an important class of lattices. Let $d$ be the dimension of $L$ and $s$ the signature. For each $(d, s) \in \mathbf{Z}_{+}$, there exists an odd unimodular non-singular lattice, which is unique if $L$ is not positive definite and written $I_{m, n}$, whereas there exists an even unimodular non-singular lattice if and only if 8 divides $s$. When $s \neq 0$, it is unique and written $I I_{m, n}$ (where $d=m+n$ and $s=m-n)$.

Suppose $L$ is even positive definite unimodular. If $d=8, L=E_{8}$; and if $d=16$, $L=E_{8} \oplus E_{8}$ or $L=D_{16}^{+}$. When $d=24$, there are 24 possibilities for $L$, all but one of which contain roots. They are called Niemeier lattices after the person who discovered them. The Leech lattice $\Lambda$ is the unique 24-dimensional even positive definite unimodular lattice with no roots, the minimal norm of an element being 4 . This lattice was first found by Leech in 1965. A simpler proof of its existence was given by Venkov [Ven] in 1975, and the first proof of its uniqueness was given by Conway ConS. In 1985 Borcherds gave a simpler proof Bor1], based on results of Venkov and of Conway and Sloane on sphere packings ConS].

If $L$ has dimension $s+2$ and signature $s$, then it is said to be a Lorentzian lattice. Then maximal negative definite subspaces of $L \otimes \mathbf{R}$ have dimension 1 , and the negative norm vectors form two open cones. Such lattices are closely linked to hyperbolic spaces. As in the positive definite case, for Lorentzian lattices, one can define the root system as the set of roots, the Weyl chambers as the regions separated out by the hyperplanes orthogonal to the roots system. Selecting a Weyl chamber $W$, one can define the simple roots as the elements having inner product at most 0 with the vectors of $W$ and orthogonal to the hyperplanes corresponding to $W$. We can therefore also define a Dynkin diagram in the usual way. An important property of the even unimodular Lorentzian lattice of dimension 26, which is, as we saw, the lattice of the roots of the fake Monster Lie algebra, is its connection with the Leech lattice: $I I_{25,1}=\Lambda \oplus I I_{1,1}$ [ConS].

6.2. Lattices and modular forms. It is well-known that theta functions provide a close connection between positive definite lattices and modular forms since $\Theta_{L}$ (see $\S 4.4$ for definition) is a modular form when $L$ is positive definite. For example,

$$
\Theta_{E_{8}}(\tau)=1+240 \sum_{n \geq 1} \sigma_{3}(n) q^{n}
$$

and

$$
\Theta_{\Lambda}(\tau)=\frac{65520}{691} \sum_{n \geq 0}\left(\sigma_{11}(n)-\tau(n)\right) q^{n}, \quad \text { where } \quad \sigma_{m}(n)=\sum_{d \mid n, d>0} d^{m}
$$


and the Ramanujan $\tau$ function is defined as follows:

$$
\sum_{n \geq 0} \tau(n) q^{n}=q \prod_{n \geq 1}\left(1-q^{n}\right)^{24} .
$$

In the more general case of a non-singular indefinite lattice $L$, the (Siegel) theta function $\theta_{L}$, generalizing the theta function of a positive definite lattice, is defined as follows. Let $V^{+}$be a maximal positive definite subspace of $L \otimes \mathbf{R}$ and $V^{-}$its negative definite complement in $L \otimes \mathbf{R}$. For $x \in L \otimes \mathbf{R}, x_{+}$and $x_{-}$will denote the projections in $V^{+}$and $V^{-}$respectively. Then

$$
\theta_{L}\left(\tau, V^{+}\right)=\sum_{x \in L} e^{2 \pi i\left(\tau x_{+}^{2} / 2+\bar{\tau} x_{-}^{2} / 2\right)}
$$

for $\tau \in \mathcal{H}$. One can define a more general vector valued theta function $\Theta_{M}$ for a lattice $M$ which can be shown to be a modular form Bor10: Set

$$
\theta_{M+\gamma}\left(\tau,(M+\gamma)^{+}\right)=\sum_{\lambda \in M+\gamma} e^{2 \pi i\left(\tau \lambda_{+}^{2} / 2+\bar{\tau} \lambda_{-}^{2} / 2\right)}
$$

for all $\gamma \in M^{\prime} / M$. Then

$$
\Theta_{M}(\tau)=\sum_{\gamma \in M^{\prime} / M} \theta_{M+\gamma} e_{\gamma}
$$

is a modular form of weight $\left(s^{+} / 2, s^{-} / 2\right)$ and type $\rho_{M}$ (defined in $\S 4.4$ ). As before the vectors $e_{\gamma}, \gamma \in M^{\prime} / M$, form a basis of $M^{\prime} / M$, and $M \otimes \mathbf{R}$ is the direct sum of a positive definite subspace of dimension $s^{+}$and a negative definite subspace of dimension $s^{-}$.

We can now state the congruence Borcherds has derived for positive definite lattices Bor10. It says that the constant term of the product of the theta function of a positive definite lattice with a modular form satisfying certain conditions is divisible by 24 . In other words, it gives congruences for various linear combinations of the coefficients of the theta function.

Let $d$ be the constant term of $\theta_{K}(\tau) / \eta(\tau)^{r k K}$, where $K$ is a positive definite even unimodular lattice and as usual $\eta(\tau)=q^{\frac{1}{24}} \prod_{n>0}\left(1-q^{n}\right)$. We apply the results of $\S 4.4$ to the unimodular lattice $M=K \oplus I I_{1,1} \oplus I I_{1,1}$ and the modular function $f=\Delta(\tau)^{-\operatorname{dim} K / 24}$. We keep the notation of $\S 4$. We can deduce that $d=24(\rho, v)$ for $v=(0,-1,0) \in K \oplus I I_{1,1}$ so that $(\rho, v)$ is an integer since both $\rho$ and $v$ are in L. Therefore

$$
d \equiv 0 \quad(\bmod 24)
$$

Bor10. M. Hopkins has recently given a simpler proof of this congruence by using topological arguments Hop.

We apply this result to a Niemeier lattice $K$. Thus $K$ has rank 24 and,

$$
\theta_{K}(\tau) / \eta(\tau)^{r k K}=\sum_{x \in K} q^{(x, x) / 2-1}\left(1+\sum_{n>0} a_{n} q^{n}\right),
$$

where the constants $a_{n}$ are non-negative. So the constant term $d$ equals the number of roots of $K$. We can thus conclude that the number of roots of a Niemeier lattice is divisible by 24 , which is well known ConS.

We end this section by giving a generalization of the above result to other modular forms Bor10. Let $K$ be a positive definite even lattice of rank s, $f$ a modular form of weight $(-s / 2,0)$ and type $\overline{\rho_{K}}$, holomorphic on $\mathcal{H}$, meromorphic at the cusps 
and with integral Fourier coefficients $c_{\gamma}(m, 0)$ when $m<0$. As above consider the vector $\rho$ in $L=K \oplus I I_{1,1}$. Set $d$ to be the constant term of $N f(\tau) \Theta_{K}(\tau)$, where $N$ is the integer giving the ideal $N \mathbf{Z}$ of $\mathbf{Z}$ generated by all possible values $(x, y)$ with $x, y \in K$. It can be shown that $24 d=(\rho, v)$ for $v=(0,0, N)$ and that this inner product is an integer Bor10. So

$$
d \equiv 0 \quad(\bmod 24) .
$$

As this paper shows there are many different ideas involved in the proof of the Moonshine Conjecture. Furthermore they lead in turn to questions related to other areas. There are many other topics connected to generalized Kac-Moody algebras which we have not mentioned, for example the study of a modular version of Moonshine and a Moonshine for other sporadic groups. The interested reader is referred to works of Ryba and Borcherds $[\mathrm{Ryb}$, BorR, Bor11] in the first case and of Conway, Norton, and Borcherds in the latter [ConN], Bor7], [Nor2].

Acknowledgements. The author is very grateful to Richard Borcherds, Haynes Miller, Jim Lepowsky, Chongying Dong and the referee for their very helpful comments. She would also like to thank the I.H.E.S. and the Mehta Institute for their hospitality.

\section{REFERENCES}

[AleCMcKS] D. Alexander, C. J. Cummins, J. McKay, C. Simons, Completely replicable functions, Groups, Combinatorics and Geometry, Ed. M. W. Liebeck, J. Saxl, Cambridge University Press 1992, 87-95

[Ati] M. Atiyah, "K-theory", W.A. Benjamin Inc., New York-Amsterdam, 1967 MR 36:7130

[BenKM] G. Benkart, S.-J. Kang, D. Melville, Quantized enveloping algebras for Borcherds superalgebras, Trans. Amer. Math. Soc. 350 (1998), no. 8, 3297-3319 MR 99f:17014

[Bor1] R. E. Borcherds, The Leech lattice, Proc. R. Soc. Lond. A 398 (1985), 365-376 MR 87g:11076

[Bor2] R. E. Borcherds, Vertex algebras, Kac-Moody algebras, and the Monster, Proc. Natl. Acad. Sci. USA 83 (May 1986), 3068-3071 MR 87m:17033

[Bor3] R. E. Borcherds, Automorphism groups of Lorentzian lattices, Journal of Algebra 111 (1) (1987), 133-153 MR 89b:20018

[Bor4] R. E. Borcherds, Generalized Kac-Moody Algebras, Journal of Algebra 115 (2) (1988), 501-512 MR 89g:17004

[Bor5] R. E. Borcherds, The Monster Lie algebra, Advances in Math. 83 (1) (Sept. 1990), 30-47 MR 91k:17027

[Bor6] R. E. Borcherds, Central extensions of generalized Kac-Moody algebras, Journal of Algebra 140 (1991), 330-335 MR 92g:17031

[Bor7] R. E. Borcherds, Monstrous Moonshine and monstrous Lie superalgebras, Invent. Math. 109 (1992), 405-444 MR 94f:11030

[Bor8] R. E. Borcherds, A characterization of generalized Kac-Moody algebras, Journal of Algebra 174 (3) (1995), 1073-1079 MR 96e:17058

[Bor9] R. E. Borcherds, Automorphic forms on $O_{s+2,2}(\mathbf{R})$ and infinite products, Invent. Math. 120 (1995), 161-213 MR 96j:11067

[Bor10] R. E. Borcherds, Automorphic forms with singularities on Grassmannians, Invent. Math. 132 (1998), 491-562 MR 99c:11049

[Bor11] R. E. Borcherds, Modular moonshine III, Duke Math. J. 93 (1998) no. 1, 129-154 MR 99e:17039

[Bor12] R. E. Borcherds, Quantum vertex algebras, Preprint (1999)

[BorR] R. E. Borcherds, A. J. E. Ryba, Modular moonshine II, Duke Math. J. 83 (1996) no. 2, 435-459 MR 98b:17030

[Bur] W. Burnside, "Group theory", Cambridge University Press, 1921 
[Con] J. H. Conway, The automorphism group of the 26-dimensional even unimodular Lorentzian lattice, J. Algebra 80 (1983), 159-163 [MR 85k:11030

[ConN] J. H. Conway, S. P. Norton, Monstrous Moonshine, Bull. London Math. Soc. 11 (1979), 308-339 MR 81j:20028

[ConS] J. Conway, N. J. A. Sloane, "Sphere packings, lattices and groups", Grundlehren der Mathematischen Wissenschaten 290, Springer Verlag, New York-Berlin, 1988 MR 89a:11067

[CumN] C. J. Cummins, S. P. Norton, Monstrous Moonshine, Bull. London Math. Soc. 11 (1979), 308-339

[CumG] C. J. Cummins, T. Gannon, Modular equations and the genus zero property of moonshine functions, Invent. Math. 129 (1997), 413-443 MR 98k:11046

[Don1] C. Dong, Representations of the moonshine module vertex operator algebra, Contemporary Math. 175 (1994), 27-36 MR 95m:17021

[Don2] C. Dong, Twisted modules for vertex algebras associated with even lattices, J. Alg. 165 (1994), 90-112 MR 95i:17032

[DonL] C. Dong, J. Lepowsky, "Generalized vertex algebras and relative vertex operators", Progress in Math. 112, Birkhäuser, Boston, 1993 MR 95b:17032

[DonM] C. Dong, G. Mason, Monstrous Moonshine of higher weight, q-alg 9803116

[DonGH] C. Dong, R. L. Griess, G. Höhn, Framed vertex operator algebras, codes and the moonshine module, Comm. Math. Phys. 193 (1998), 407-408 MR 99g:17050

[DonLM1] C. Dong, H. Li, G. Mason, Twisted representations of vertex operator algebras, Math. Ann. 310 (1998) 571-600 MR 99d:17030

[DonLM2] C. Dong, H. Li, G. Mason, Twisted representations of vertex operator algebras and associative algebras, Int. Math. Res., No. 7 No. 8 (1998), 389-397 MR 99f:17032

[Dys] F. Dyson, Missed opportunities, Bull. Amer. Math. Soc. 78 No. 5 (1972), 635-652 MR 58:25442

[EicZ] M. Eichler, D. Zagier, "The theory of Jacobi forms", Progress in Math. 55, Birkhäuser, Boston, 1985 MR 86j:11043

[EtinK] P. Etingof, D. Kazhdan, Quantization of Lie bialgebras, V., Preprint math. QA/9808121

[FeinL] A. Feingold, J. Lepowsky, The Weyl-Kac character formula and power series identities, Advances in Math. 29 (1978), 271-309 MR 83a:17015

[FeitT] W. Feit, J. G. Thompson, Solvability of groups of odd order, Pacific J. Math. 13 (1963), 775-1029 MR 29:3538

[FisLT] B. Fischer, D. Livingstone, M. P. Thorne, The characters of the "Monster" simple group, Birmingham (1978)

[Fre] I. B. Frenkel, Representations of Kac-Moody algebras and dual resonance models, Lectures in Applied Mathematics 21 (1985), 325-353 MR 87b:17010

[FreJ] I. B. Frenkel, N. H. Jing, Vertex representations of quantum affine algebras, Proc. Nat. Acad. Sci. U.S.A. 85 (1988) no. 24, 9373-9377 MR 90e:17028

[FreK] I. B. Frenkel, V. G. Kac, Basic representations of affine algebras, Invent. Math. 62 (1980), 99-120 MR 84f:17004

[FreLM1] I. B. Frenkel, J. Lepowsky, J. Meurman, A natural representation of the FischerGriess Monster with the modular function $J$ as character, Proc. Natl. Acad. Sci. USA 81 (1984), 3256-3260 MR 85e:20018

[FreLM2] I. B. Frenkel, J. Lepowsky, J. Meurman, "Vertex operator algebras and the Monster", Academic Press, 1988 MR 90h:17026

[Fru] A. Frumkin, The irreducible characters of the Lie superalgebras of type $A(m, n)$ and filtrations of their Kac modules, Israel J. Math. 96 Part A (1996), 267-279 MR 98b:17006

[GabK] O. Gabber, V. G. Kac, On defining relations of certain infinite-dimensional Lie algebras, Bull. Amer. Math. Soc. 5 (1981), 185-189 MR 84b:17011

[Gar] H. Garland, Lectures on loop algebras and the Leech lattice, Yale University, spring 1980

[GarL] H. Garland, J. Lepowsky, Lie algebra homology and the Macdonald-Kac formulas, Invent. Math. 34 (1976), 37-76 MR 54:2744 
[GodT] P. Goddard, C. B. Thorn, Compatibility of the dual pomeron with unitary and the absence of ghosts in the dual resonance model, Physics Letters 40B (26 June 1972) no.2, 235-237

[Gor] D. Gorenstein, "Finite simple groups", Plenum Press, New York, 1982 MR 84j:20002

[Gri] R. L. Griess, Jr., The friendly giant, Invent. Math. 69 (1982), 1-102 MR 84m:20024

[GriN1] V. A. Gritsenko, V.V. Nikulin, Igusa modular forms and the "simplest" Lorentzian Kac-Moody algebras, Sbornik Math 187 no. 11 (1996), 1601-1641 MR 98a:11059

[GriN2] V. A. Gritsenko, V.V. Nikulin, Automorphic forms and Lorentzian Kac-Moody algebras, Part I, Intern. J. Math. 9 no. 2 (1998), 153-199 MR 99b:11040

[GriN3] V. A. Gritsenko, V.V. Nikulin, Automorphic forms and Lorentzian Kac-Moody algebras, Part II, Intern. J. Math. 9 no. 2 (1998), 201-275 MR 99b:11041

[Hop] M. J. Hopkins, On Borcherds' Theta Congruence, unpublished notes

[Jeu] J. Van der Jeugt, Character formulae for the Lie superalgebra $C(n)$, Comm. Algebra 19 (1991), 199-222. MR 92b:17050

[JeuHKT] J. Van der Jeugt, J. W. B. Hughes, R. C. King, J. Thierry-Mieg, A character formula for singly atypical modules of the Lie superalgebra $\mathrm{sl}(m / n)$, Comm. Algebra 18, no. 10 (1990), 3453-3480 MR 91j:17046

[JorT] J. Jorgenson, A. Todorov, Enriquez surfaces, analytic discriminants and Borcherds' phi function, Comm. Math. Phys. 191 (1998), 249-264 MR 99e:14042

[Jur] E. Jurisich, Generalized Kac-Moody Lie algebras, free Lie algebras and the structure of the Monster Lie algebra J. Pure Appl. Algebra 122 (1997), 233-266 MR 99b:17032

[JurLW] E. Jurisich, J. Lepowsky, R. L. Wilson, Realizations of the Monster Lie algebra, Selecta Mathematica, New Series 1 (1995), 129-161 MR 96e:17059

[Kac1] V.G. Kac, Simple irreducible graded Lie algebras of finite growth, (English translation) Math. USSR-Izvestija 2 (1968), 1271-1311 MR 41:4590

[Kac2] V.G. Kac, Infinite-dimensional Lie algebras and Dedekind's $\eta$-function, (English translation) Funct. Anal. Appl. 8 (1974), 68-70 MR 51:10410

[Kac3] V. G. Kac, Lie Superalgebras, Advances in Mathematics 26 (1977), 8-96 MR 58:5803

[Kac4] V. G. Kac, Infinite-Dimensional Algebras, Dedekind's $\eta$-Function, Classical Möbius Function and the Very Strange Formula, Advances in Mathematics 30 (1978), 85136 MR 83a:17014a

[Kac5] V. G. Kac, "Representations of classical Lie superalgebras", Lecture Notes in Math. 676 (1978), 597-626 MR 80f:17006

[Kac6] V. G. Kac, An elucidation of "infinite-dimensional ... and the very strange formula" $E_{8}^{(1)}$ and the cube root of the modular invariant $j$, Advances in Math. 35 (1980), 264-273 MR 83a:17014b

[Kac7] V. G. Kac, On simplicity of certain infinite-dimensional Lie algebras, Bull. Amer. Math. Soc. 2 (1980), 311-314 MR 82a:17008

[Kac8] V. G. Kac, A remark on the Conway-Norton conjecture about the "Monster" simple group, Proc. Natl. Acad. Sci. USA 77 (1980), 5048-5049 MR 81m:20026

[Kac9] V. G. Kac, "Infinite dimensional Lie algebras", third ed., Cambridge University Press, 1990 MR 92k:17038

[Kac10] V. G. Kac, "Vertex algebras for beginners", University Lecture Series, 10. American Mathematical Society, Providence, RI, 1997 MR 99a:17027

[KacK] V. G. Kac, S.-J. Kang, Trace formula for graded Lie algebras and Monstrous Moonshine, Canad. Math. Soc. Conf. Proc. 16 (1995), 141-14 MR 96k:17044

[KacKLW] V. G. Kac, D. A. Kazhdan, J. Lepowsky, R. L. Wilson, Realization of the basic representation of the Euclidean Lie algebras, Advances in Math. 42 (1981), 83-112 MR 83a:17016

[KacP1] V. G. Kac, D. H. Peterson, Affine Lie algebras and Hecke modular forms, Bull. Amer. Math. Soc. 3 (1980), 1057-1061 MR 82b:10028

[KacP2] V. G. Kac, D. H. Peterson, Infinite flag varieties and conjugacy theorems, Proc. Natl. Acad. Sci., USA 80 (1983), 1778-1782 MR 84g:17017

[KacP3] V. G. Kac, D. H. Peterson, Infinite-dimensional Lie algebras, theta functions and modular forms, Advances in Math. 53 (1984), 125-264 MR 86a:17007 
[KacP4] V. G. Kac, D. H. Peterson, Unitary structure in representations of infinitedimensional groups and a convexity theorem, Invent. Math. 76 (1984), 1-14 MR 86g:17013

[KacP5] V. G. Kac, D. H. Peterson, 112 constructions of the basic representation of the loop group of $E_{8}$, Proceedings of the conference "Anomalies, geometry, topology" Argonne, 1985. World Sci., 1985, 276-298 MR 88c:17022

$[\mathrm{KacW}] \quad$ V. G. Kac, W. Wakimoto, Integrable highest weight modules over affine Lie superalgebras and number theory, Progress in Math. 123 (1994), 415-456 MR 96j:11056

[Kan1] S.-J. Kang, Root multiplicities of Kac-Moody algebras, Duke Math J. 74 (1994), 635-666 MR 95c:17036

[Kan2] S.-J. Kang, Generalized Kac-Moody algebras and the modular function $j$, Math. Ann. 298 (1994), 373-384 MR 94m:17026

[Kan3] S.-J. Kang, Quantum deformations of generalized Kac-Moody algebras and their modules, J. Algebra 175 (1995) no. 3, 1041-1066 MR 96k:17023

[Koi] M. Koike, On replication formula and Hecke operators, Nagoya University preprint

[Kum] S. Kumar, Demazure character formula in arbitrary Kac-Moody setting, Invent. Math. 89 (1987), 395-423 MR 88i:17018

[Lep1] J. Lepowsky, Euclidean Lie algebras and the modular function $j$, in: Santa Cruz Conference ion Finite Groups, 1979, Proc. Symp. Pure Math., American Math. Soc. 37 (1980), 567-570 MR 82h:17010

[Lep2] J. Lepowsky, Calculus of twisted vertex operators, Proc. Natl. Acad. Sci. USA. 82 (1985), 8295-8299 MR 88f:17030

[LepW] J. Lepowsky, R. L. Wilson, Construction of the affine Lie algebra $A_{1}^{(1)}$, Commun. Math. Phys. 62 (1978), 43-53 MR 58:28089

[Leu] J. W. van de Leur, A classification of contragredient Lie superalgebras of finite growth, Communications in Algebra 17 (1989), 1815-1841 MR 90j:17008

[Li] H.-S. Li, Local systems of vertex operators, vertex superalgebras and modules, $J$. Pure Appl. Algebra 109 (1996), 143-195 MR 97d:17016

[Liu] L.-S. Liu, Kostant's formula in Kac-Moody algebras, J. Algebra 149 (1992), 155-178 MR 93d:17011

[Mac] I. G. Macdonald, Affine root systems and Dedekind's $\eta$-function, Invent. Math. 15 (1972), 91-143 MR 50:9996

[Man] S. Mandelstam, Dual resonance models, Phys. Rep. 13 (1974), 259-353 MR 57:18559

[Mat1] O. Mathieu, Formule de Demazure-Weyl et théorème de Borel-Weil-Bott pour les algèbres de Kac-Moody générales, C.R. Acad. Sci. Paris 303 (1986), 391-394 MR 87m:17036

[Mat2] O. Mathieu, Classification of simple graded Lie algebras of finite growth, Invent. Math. 108 (1992), 455-519 MR 93h:17069

[Moo] R. V. Moody, A new class of Lie algebras, J. Algebra 10 (1968), 211-230 MR 37:5261

[Nai] S. Naito, The strong Bernstein-Gel'fand-Gel'fand resolution for generalized KacMoody algebras. I, Publ. RIMS 29 (1993), 709-730 MR 94k:17040

[Nik1] V. V. Nikulin, A lecture on Kac-Moody Lie algebras of the arithmetic type, Preprint Queen's University, Canada 1994-16 (1994), alg-geom 9412003

[Nik2] V. V. Nikulin, A theory of Lorentzian Kac-Moody algebras, alg-geom 9810001

[Nor1] S. P. Norton, More on Moonshine, Computational group theory, Academic Press (1984), 185-193 MR 86h:20020

[Nor2] S. P. Norton, Generalized moonshine, Proc. Symp. Pure Math. 47 (1987), 208-209 MR 89c:11066

[Ray1] U. Ray, A character formula for generalized Kac-Moody superalgebras, J. Algebra 177 (1995), 154-163 MR 97f:17008

[Ray2] U. Ray, Some subalgebras of the Monster Lie algebra and the modular polynomial, J. Algebra 191 (1997), 109-126 MR 98g:17028

[Ray3] U. Ray, A Characterization Theorem for a certain class of graded Lie superalgebras, J. Algebra, to appear

[Ryb] A. J. E. Ryba, Modular Moonshine?, Contemp. Math., vol. 193, Amer. Math. Soc., Providence, RI, 1996. MR 97c:20022 
[Sch] N. R. Scheithauer, The fake monster superalgebra, Adv. Math. 151 (2000), 226-269 CMP 2000:12

[Seg] G. Segal, Unitary representations of some infinite-dimensional groups, Comm. Math. Phys. 80 (1981), 301-342 MR 82k:22004

[Serg] V. Serganova, Kazhdan-Lusztig polynomials and character formula for the Lie superalgebra $\mathfrak{g l}(m \mid n)$, Selecta Math. (N.S.) 2 no. 4 (1996), 607-651 MR 98f:17007

[Ser] J.-P. Serre, "Algèbres de Lie semisimples complexes", W. A. Benjamin, New York, 1966 MR 35:6721

[Shi] G. Shimura, "Introduction to the arithmetic theory of automorphic functions", Princeton University Press, Princeton, New Jersey, 1971 MR 47:3318

[Tits] J. Tits, On R. Griess' "Friendly Giant", Invent. Math. 78 (1984), 491-499 MR 86f:20019

[Ven] B. B. Venkov, On the classification of integral even unimodular 24-dimensional quadratic forms, Proc. Steklov Inst. Math. 4 (1980), 63-74 MR 81d:10024

[Vin] E. B. Vinberg, Some arithmetical discrete groups in Lobachevskii spaces, in "Discrete subgroups of Lie groups", Oxford University Press, 1975 323-348 MR 54:10492

Université DE Reims, France

E-mail address: ray@ihes.fr 